\title{
Scalable Proximity Estimation and Link Prediction in Online Social Networks
}

\author{
Han Hee Song Tae Won Cho Vacha Dave Yin Zhang Lili Qiu \\ The University of Texas at Austin \\ \{hhsong, khatz, vacha, yzhang, lili\}@cs.utexas.edu
}

\begin{abstract}
Proximity measures quantify the closeness or similarity between nodes in a social network and form the basis of a range of applications in social sciences, business, information technology, computer networks, and cyber security. It is challenging to estimate proximity measures in online social networks due to their massive scale (with millions of users) and dynamic nature (with hundreds of thousands of new nodes and millions of edges added daily). To address this challenge, we develop two novel methods to efficiently and accurately approximate a large family of proximity measures. We also propose a novel incremental update algorithm to enable near real-time proximity estimation in highly dynamic social networks. Evaluation based on a large amount of real data collected in five popular online social networks shows that our methods are accurate and can easily scale to networks with millions of nodes.

To demonstrate the practical values of our techniques, we consider a significant application of proximity estimation: link prediction, i.e., predicting which new edges will be added in the near future based on past snapshots of a social network. Our results reveal that (i) the effectiveness of different proximity measures for link prediction varies significantly across different online social networks and depends heavily on the fraction of edges contributed by the highest degree nodes, and (ii) combining multiple proximity measures consistently yields the best link prediction accuracy.
\end{abstract}

\section{Categories and Subject Descriptors}

H.3.5 [Information Storage and Retrieval]: Online Information Services-Web-based services; J.4 [Computer Applications]: Social and Behavioral Sciences-Sociology

\section{General Terms}

Algorithms, Human Factors, Measurement

\section{Keywords}

Social Network, Proximity Measure, Link Prediction, Embedding, Matrix Factorization, Sketch

Permission to make digital or hard copies of all or part of this work for personal or classroom use is granted without fee provided that copies are not made or distributed for profit or commercial advantage and that copies bear this notice and the full citation on the first page. To copy otherwise, to republish, to post on servers or to redistribute to lists, requires prior specific permission and/or a fee.

IMC'09, November 4-6, 2009, Chicago, Illinois, USA.

Copyright 2009 ACM 978-1-60558-770-7/09/11 ...\$10.00.

\section{INTRODUCTION}

A social network [53] is a social structure modeled as a graph, where nodes represent people or other entities embedded in a social context, and edges represent specific types of interdependency among entities, e.g., values, visions, ideas, financial exchange, friendship, kinship, dislike, conflict or trade. Understanding the nature and evolution of social networks has important applications in a number of fields such as sociology, anthropology, biology, economics, information science, and computer science.

Traditionally, studies on social networks often focus on relatively small social networks (e.g., [30,31] examine co-authorship networks with about 5000 nodes). Recently, however, social networks have gained tremendous popularity in the cyber space. Online social networks such as MySpace [40], Facebook [18] and YouTube [55] have each attracted tens of millions of visitors every month [44] and are now among the most popular sites on the Web [4]. The wide variety of online social networks and the vast amount of rich information available in these networks represent an unprecedented research opportunity for understanding the nature and evolution of social networks at massive scale.

A central concept in the computational analysis of social networks is proximity measure, which quantifies the closeness or similarity between nodes in a social network. Proximity measures form the basis for a wide range of important applications in social and natural sciences (e.g., modeling complex networks [6, 13, 25, 42]), business (e.g., viral marketing [23], fraud detection [11]), information technology (e.g., improving Internet search [35], collaborative filtering [7]), computer networks (e.g., constructing overlay networks [45]), and cyber security (e.g., mitigating email spams [22], defending against Sybil attacks [56]).

Unfortunately, the explosive growth of online social networks imposes significant challenges on proximity estimation. First, online social networks are typically massive in scale. For example, MySpace has over 400 million user accounts [41], and Facebook has reportedly over 120 million active users world wide [19]. As a result, many proximity measures that are highly effective in relatively small social networks (e.g., the classic Katz measure [26]) become computationally prohibitive in large online social networks with millions of nodes [48]. Second, online social networks are often highly dynamic, with hundreds of thousands of new nodes and millions of edges added daily. In such fast-evolving social networks, it is challenging to compute up-to-date proximity measures in a timely fashion.

Approach and contributions. To address the above challenges, we develop two novel techniques, proximity sketch and proximity embedding, for efficient and accurate proximity estimation in large social networks with millions of nodes. We then augment these techniques with a novel incremental proximity update algorithm to enable near real-time proximity estimation in highly dynamic 
social networks. Our techniques are applicable to a large family of commonly used proximity measures, which includes the aforementioned Katz measure [26], as well as rooted PageRank [30,31] and escape probability [50]. These proximity measures are known to be highly effective for many applications $[30,31,50]$, but were previously considered computationally prohibitive for large social networks $[48,50]$.

To demonstrate the practical value of our techniques, we consider a significant application of proximity estimation: link prediction, which refers to the task of predicting the edges that will be added to a social network in the future based on past snapshots of the network. As shown in [30,31], proximity measures lie right at the heart of link prediction. Understanding which proximity measures lead to the most accurate link predictions provides valuable insights into the nature of social networks and can serve as the basis for comparing various network evolution models (e.g., $[6,13,25,42])$. Accurate link prediction also allows online social networks to automatically make high-quality recommendations on potential new friends, making it much easier for individual users to expand their social neighborhood.

We evaluate the effectiveness of our proximity estimation methods using a large amount of real data collected in five popular online social networks: Digg [14], Flickr [20], LiveJournal [33], MySpace [40], and YouTube [55]. Our results show that our methods are accurate and can easily scale to handle large social networks with millions of nodes and hundreds of millions of edges. We also conduct extensive experiments to compare the effectiveness of a variety of proximity measures for link prediction in these online social networks. Our results uncover two interesting new findings: (i) the effectiveness of different proximity measures varies significantly across different networks and depends heavily on the fraction of edges contributed by the highest degree nodes, and (ii) combining multiple proximity measures using an off-the-shelf machine learning software package consistently yields the best link prediction accuracy.

Paper organization. The rest of the paper is organized as follows. In Section 2, we develop techniques to efficiently and accurately approximate a large family of proximity measures in massive, dynamic online social networks. In Section 3, we describe link prediction techniques. In Section 4, we evaluate both proximity estimation and link prediction in five popular online social networks. In Section 5, we review related work. We conclude in Section 6.

\section{SCALABLE PROXIMITY ESTIMATION}

Proximity measures are the basis for many applications of social networks. As a result, a variety of proximity measures have been proposed. The simplest proximity measures are based on either the shortest graph distance or the maximum information flow between two nodes. One can also define proximity measures based on node neighborhoods (e.g., the number of common neighbors). Finally, several more sophisticated proximity measures involve infinite sums over the ensemble of all paths between two nodes (e.g., Katz measure [26], rooted PageRank [30,31], and escape probability [50]). Compared with more direct proximity measures such as shortest graph distances and numbers of shared neighbors, pathensemble based proximity measures capture more information about the underlying social structure and have been shown to be more effective in social networks with thousands of nodes [30,31,50].

Despite the effectiveness of path-ensemble based proximity measures, it is computationally expensive to summarize the ensemble of all paths between two nodes. The state of the art in estimating path-ensemble based proximity measures (e.g., [50]) typically can only handle social networks with tens of thousands of nodes. As a result, recent works on proximity estimation in large social networks (e.g., [48]) either dismiss path-ensemble based proximity measures due to their prohibitive computational cost or leave it as future work to compare with these proximity measures.

In this section, we address the above challenge by developing efficient and accurate techniques to approximate a large family of path-ensemble based proximity measures. Our techniques can handle social networks with millions of nodes, which are several orders of magnitude larger than what the state of the art can support. In addition, our techniques can support near real-time proximity estimation in highly dynamic social networks.

\subsection{Problem Formulation}

Below we first formally define three commonly used path-ensemble based proximity measures: (i) Katz measure, (ii) rooted PageRank, and (iii) escape probability. We then show that all three proximity measures can be efficiently estimated by solving a common subproblem, which we term the proximity inversion problem. In all our discussions below, we model a social network as a graph $G=(V, E)$, where $V$ is the set of nodes, and $E$ is the set of edges. $G$ can be either undirected or directed, depending on whether the social relationship is symmetric.

Katz measure. The Katz measure [26] is a classic path-ensemble based proximity measure. It is designed to capture the following simple intuition: the more paths there are between two nodes and the shorter these paths are the stronger the relationship is (because there are more opportunities for the two nodes to discover and interact with each other in the social network). Given two nodes $x, y \in V$, the Katz measure $\operatorname{Katz}[x, y]$ is a weighted sum of the number of paths from $x$ to $y$, exponentially damped by length to count short paths more heavily. Formally, we have

$$
\operatorname{Katz}[x, y]=\sum_{\ell=1}^{\infty} \beta_{\mathrm{Katz}}^{\ell} \cdot\left|\operatorname{paths}_{x, y}^{\langle\ell\rangle}\right|
$$

where paths $x_{x, y}^{\langle\ell\rangle}$ is the set of length- $\ell$ paths from $x$ to $y$, and $\beta_{\mathrm{Katz}}$ is a damping factor.

Let $A$ be the adjacency matrix of graph $G$, where

$$
A[x, y]= \begin{cases}1, & \text { if }\langle x, y\rangle \in E \\ 0, & \text { otherwise }\end{cases}
$$

As shown in [31], the Katz measures between all pairs of nodes (represented as a matrix Katz) can be derived as a function of the adjacency matrix $A$ and the damping factor $\beta_{\text {Katz }}$ as follows:

$$
\text { Katz }=\sum_{\ell=1}^{\infty} \beta_{\mathrm{Katz}}^{\ell} A^{\ell}=\left(I-\beta_{\mathrm{Katz}} A\right)^{-1}-I
$$

where $I$ is the identity matrix. Thus, in order to compute Katz, we just need to compute the matrix inverse $\left(I-\beta_{\mathrm{Katz}} A\right)^{-1}$.

Rooted PageRank. The rooted PageRank [30,31] is a special instance of personalized PageRank [8,12]. It defines a random walk on the underlying graph $G=(V, E)$ to capture the probability for two nodes to run into each other and uses this probability as an indicator of the node-to-node proximity. Specifically, given two nodes $x, y \in V$, the rooted PageRank $\operatorname{RPR}[x, y]$ is defined as the stationary probability of $y$ under the following random walk: (i) with probability $1-\beta_{\mathrm{RPR}}$, jump to node $x$, and (ii) with probability $\beta_{\mathrm{RPR}}$, move to a random neighbor of current node.

The rooted PageRank between all node pairs (represented as a matrix RPR) can be derived as follows. Let $D$ be a diagonal matrix with $D[i, i]=\sum_{j} A[i, j]$. Let $T=D^{-1} A$ be the adjacency matrix with row sums normalized to 1 . We then have:

$$
\mathrm{RPR}=\left(1-\beta_{\mathrm{RPR}}\right)\left(I-\beta_{\mathrm{RPR}} T\right)^{-1}
$$


Therefore, to compute RPR, we just need to compute the matrix inverse $\left(I-\beta_{\mathrm{RPR}} T\right)^{-1}$. Also note that the standard PageRank can be computed simply as the average of all the columns of RPR.

Escape probability. The escape probability [50] is another pathensemble based proximity measure. Given two nodes $x, y \in V$, the escape probability $\operatorname{EP}[x, y]$ from $x$ to $y$ is defined as the probability that a random walk which starts from node $x$ will visit node $y$ before it returns to node $x$ [16]. The escape probability EP $[x, y]$ can be directly derived from the rooted PageRank as follows.

$$
\operatorname{EP}[x, y]=\frac{Q[x, y]}{Q[x, x] Q[y, y]-Q[x, y] Q[y, x]}
$$

where matrix $Q=\operatorname{RPR} /\left(1-\beta_{\mathrm{RPR}}\right)=\left(I-\beta_{\mathrm{RPR}} T\right)^{-1}$.

As shown in [16], when the underlying graph $G=(V, E)$ is undirected, the escape probability EP is also closely related to several other random walk induced proximity or distance measures: effective conductance EC, effective resistance ER, and commute time CT. Specifically, we have:

$$
\begin{aligned}
\mathrm{EC}[x, y] & =|N(x)| \cdot \operatorname{EP}[x, y] \\
\operatorname{ER}[x, y] & =1 / \operatorname{EC}[x, y] \\
\mathrm{CT}[x, y] & =2 \cdot|E| \cdot \operatorname{ER}[x, y]
\end{aligned}
$$

The common subproblem: proximity inversion. From the above discussions, it is evident that the key to estimating all three pathensemble based proximity measures is to efficiently compute elements of the following matrix inverse:

$$
P \triangleq(I-\beta M)^{-1}=\sum_{\ell=0}^{\infty} \beta^{\ell} M^{\ell}
$$

where $M$ is a sparse nonnegative matrix with millions of rows and columns, $I$ is an identity matrix of the same size, and $\beta \geq 0$ is a damping factor. We term this common subproblem the proximity inversion problem.

\subsection{Scalable Proximity Inversion}

The key challenge in solving the proximity inversion problem (i.e., computing elements of matrix $P=(I-\beta M)^{-1}$ ) is that while $M$ is a sparse matrix, $P$ is a dense matrix with millions of rows and columns. It is thus computationally prohibitive to compute and/or store the entire $P$ matrix. To address the challenge, we first develop two novel dimensionality reduction techniques to approximate elements of $P=(I-\beta M)^{-1}$ based on a static snapshot of $M$ : proximity sketch and proximity embedding. We then develop an incremental proximity update algorithm to approximate elements of $P$ in an online setting when $M$ continuously evolves.

\subsubsection{Preparation}

We first present an algorithm to approximate the sum of a subset of rows or columns of $P=(I-\beta M)^{-1}$ efficiently and accurately. We use this algorithm as a basic building block in both proximity sketch and proximity embedding.

Algorithm. Suppose we want to compute the sum of a subset of columns: $\sum_{i \in S} P[*, i]$, where $S$ is a set of column indices. We first construct an indicator column vector $v$ such that $v[i]=1$ for $\forall i \in S$ and $v[j]=0$ for $\forall j \notin S$. The sum of columns $\sum_{i \in S} P[*, i]$ is simply $P v$ and can be approximated as:

$$
P v=(I-\beta M)^{-1} v=\sum_{\ell=0}^{\infty} \beta^{\ell} M^{\ell} v \approx \sum_{\ell=0}^{\ell_{\max }} \beta^{\ell} M^{\ell} v
$$

where $\ell_{\max }$ bounds the maximum length of the paths over which the summation is performed.

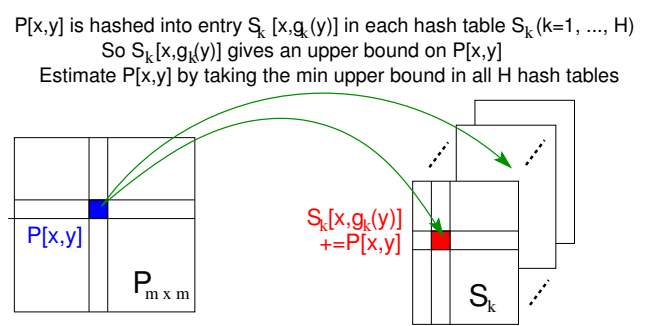

Figure 1: Proximity sketch

Similarly, to compute the sum of a subset of rows $\sum_{i \in S} P[i, *]$, we first construct an indicator row vector $u$ such that $u[i]=1$ for $\forall i \in S$ and $u[j]=0$ for $\forall j \notin S$. We then approximate the sum of rows $\sum_{i \in S} P[i, *]=u P$ as:

$$
u P=u(I-\beta M)^{-1}=\sum_{\ell=0}^{\infty} \beta^{\ell} u M^{\ell} \approx \sum_{\ell=0}^{\ell_{\max }} \beta^{\ell} u M^{\ell}
$$

In one extreme where $S$ contains all the column indices, we can compute the sum of all columns in $P$. This is useful for computing the PageRank (which is the average of all columns in the RPR matrix). In the other extreme where $S$ contains only one element, we can efficiently approximate a single row or column of $P$.

Complexity. Suppose $M$ is an $m$-by- $m$ matrix with $n$ non-zeros. Computing the product of sparse matrix $M$ and a dense vector $v$ takes $O(n)$ time by exploiting the sparseness of $M$. So it takes $O\left(n \cdot \ell_{\max }\right)$ time to compute $\left\{M^{\ell} v \mid \ell=1, \ldots, \ell_{\max }\right\}$ and approximate $P v$. Note that the time complexity is independent of the size of the subset $S$. The complexity for computing $u P$ is identical.

Note however that the above approximation algorithm is not efficient for estimating individual elements of $P$. In particular, even if we only want a single element $P[x, y]$, we have to compute either a complete row $P[x, *]$ or a complete column $P[*, y]$ in order to obtain an estimate of $P[x, y]$. So we only apply the above technique for preprocessing. We will develop several techniques in the rest of this section to estimate individual elements of $P$ efficiently.

Benefits of truncation. We achieve two key benefits by truncating the infinite expansion $\sum_{\ell=0}^{\infty} \beta^{\ell} M^{\ell}$ to form a finite expansion $\sum_{\ell=0}^{\ell_{\max }} \beta^{\ell} M^{\ell}$. First, we completely eliminate the influence of paths with length above $\ell_{\max }$ on the resulting sums. This is desirable because as pointed out in [30,31], proximity measures that are unable to limit the influence of overly lengthy paths tend to perform poorly for link prediction. Second, we ensure that $\sum_{\ell=0}^{\ell_{\max }} \beta^{\ell} M^{\ell}$ is always finite, whereas elements of $\sum_{\ell=0}^{\infty} \beta^{\ell} M^{\ell}$ may reach infinity when the damping factor $\beta$ is not small enough.

\subsubsection{Proximity Sketch}

Our first dimensionality reduction technique, proximity sketch, exploits the mice-elephant phenomenon that frequently arises in matrix $P$ in practice, i.e., most elements in $P$ are tiny (i.e., mice) but few elements are huge (i.e., elephants).

Algorithm. Figure 1 shows the data structure for our proximity sketch, which consists of $H$ hash tables: $S_{1}, \cdots, S_{H}$. Each $S_{k}$ is a 2-dimensional array with $m$ rows and $c \ll m$ columns. A column hash function $g_{k}:\{1, \cdots, m\} \rightarrow\{1, \cdots, c\}$ is used to hash each element in $P_{m \times m}(P[x, y])$ into an element in $S_{k} m \times c$ $\left(S_{k}\left[x, g_{k}(y)\right]\right)$. We ensure that different hash functions $g_{k}(\cdot)(k=$ $1, \cdots, H)$ are two-wise independent. In each $S_{k}$, each element $P[x, y]$ is added to entry $S_{k}\left[x, g_{k}(y)\right]$. Thus,

$$
S_{k}[a, b]=\sum_{y: g_{k}(y)=b} P[a, y]
$$


Note that each column of $S_{k}: S_{k}[*, b]=\sum_{y: g_{k}(y)=b} P[*, y]$ can be computed efficiently as described in Section 2.2.1.

Since $P$ is a nonnegative matrix, for any $x, y \in V$ and any $k \in$ $[1, H], S_{k}\left[x, g_{k}(y)\right]$ is an upper bound for $P[x, y]$ according to Eq. 12. We can therefore estimate $P[x, y]$ by taking the minimum upper bound in all $H$ hash tables in $O(H)$ time. That is:

$$
\hat{P}[x, y]=\min _{k} S_{k}\left[x, g_{k}(y)\right]
$$

Probabilistic accuracy guarantee. Our proximity sketch effectively summarizes each row of $P: P[x, *]$ using a count-min sketch [10]: $\left\{S_{k}[x, *] \mid k=1, \cdots, H\right\}$. As a result, we provide the same probabilistic accuracy guarantee as the count-min sketch, which is summarized in the following theorem (see [10] for detailed proof).

THEOREM 1. With $H=\left\lceil\ln \frac{1}{\delta}\right\rceil$ hash tables, each with $c=\left\lceil\frac{\mathrm{e}}{\epsilon}\right\rceil$ columns, the estimate $\hat{P}[x, y]$ guarantees: (i) $P[x, y] \leq \hat{P}[x, y]$; and (ii) with probability at least $1-\delta, \hat{P}[x, y] \leq P[x, y]+\epsilon$. $\sum_{z} P[x, z]$.

Therefore, as long as $P[x, y]$ is much larger than $\epsilon \cdot \sum_{z} P[x, z]$, the relative error of $\hat{P}[x, y]$ is small with high probability.

Extension. If desired, we can further reduce the space requirement of proximity sketch by aggregating the rows of $S_{k}$ (at the cost of lower accuracy). Specifically, we associate each $S_{k}$ with a row hash function $f_{k}(\cdot)$. We then compute

$$
R_{k}[a, b]=\sum_{x: f_{k}(x)=a} S_{k}[x, b]
$$

and store $\left\{R_{k}\right\}$ (instead of $\left\{S_{k}\right\}$ ) as the final proximity sketch. Clearly, we have $R_{k}[a, b]=\sum_{x: f_{k}(x)=a} \sum_{y: g_{k}(y)=b} P[x, y]$. For any $x, y \in V$, we can then estimate $P[x, y]$ as

$$
\hat{P}[x, y]=\min _{k} R_{k}\left[f_{k}(x), g_{k}(y)\right]
$$

\subsubsection{Proximity Embedding}

Our second dimensionality reduction technique, proximity embedding, applies matrix factorization to approximate $P$ as the product of two rank- $r$ factor matrices $U$ and $V$ :

$$
P_{m \times m} \approx U_{m \times r} \cdot V_{r \times m}
$$

In this way, with $O(2 m r)$ total state for factor matrices $U$ and $V$, we can approximate any $P[x, y]$ in $O(r)$ time as:

$$
\hat{P}[x, y]=\sum_{k=1}^{r} U[x, k] \cdot V[k, y]
$$

Our technique is motivated by recent research on embedding network distance (e.g., end-to-end round-trip time) into low-dimensional space (e.g., $[32,34,43,49])$. Note however that proximity is the opposite of distance - the lower the distance the higher the proximity. As a result, techniques effective for distance embedding do not necessarily work well for proximity embedding.

Algorithm. As shown in Figure 2(a), our goal is to derive the two rank- $r$ factor matrices $U$ and $V$ based on only a subset of rows $P[L, *]$ and columns $P[*, L]$, where $L$ is a set of indices (which we term the landmark set). We achieve this goal by taking the following five steps:

1. Randomly select a subset of $\ell$ nodes as the landmark set $L$. The probability for a node $i$ to be included in $L$ is proportional to the PageRank of node $i$ in the underlying graph ${ }^{1}$. Note that

\footnotetext{
${ }^{1}$ We also consider uniform landmark selection, but it yields worse accuracy than PageRank based landmark selection (see Section 4).
}

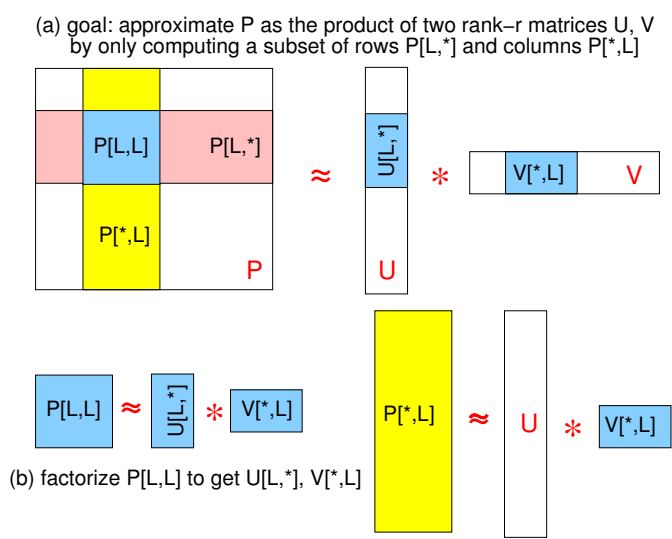

(c) obtain $\mathrm{U}$ from $\left.\mathrm{P}{ }^{*}, \mathrm{~L}\right]$ and $\mathrm{V}\left[{ }^{*}, \mathrm{~L}\right]$

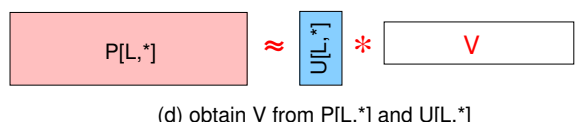

(d) obtain $\mathrm{V}$ from $\mathrm{P}\left[\mathrm{L},{ }^{*}\right]$ and $\mathrm{U}\left[\mathrm{L},{ }^{*}\right]$

Figure 2: Proximity embedding

the PageRank for all the nodes can be precomputed efficiently using the finite expansion method in Section 2.2.1.

2. Compute sub-matrices $P[L, *]$ and $P[*, L]$ efficiently by computing each row $P[i, *]$ and each column $P[*, i](i \in L)$ separately as described in Section 2.2.1.

3. As shown in Figure 2(b), apply singular value decomposition (SVD) to obtain the best rank- $r$ approximation of $P[L, L]$ :

$$
P[L, L] \approx U[L, *] \cdot V[*, L]
$$

4. Our goal is to find $U$ and $V$ such that $U \cdot V$ is a good approximation of $P$. As a result, $U \cdot V[*, L]$ should be a good approximation of $P[*, L]$. We can therefore find $U$ such that $U$. $V[*, L]$ best approximates sub-matrix $P[*, L]$ in least-squares sense (shown in Figure 2(c)). Given the use of SVD in step 3, the best $U$ is simply

$$
U=P[*, L] \cdot V[*, L]^{T}
$$

5. Similarly, find $V$ such that $U[L, *] \cdot V$ best approximates submatrix $P[L, *]$ in least-squares sense (shown in Figure 2(d)), which is simply

$$
V=U[L, *]^{T} \cdot P[L, *]
$$

Accuracy. Unlike proximity sketch, proximity embedding does not provide any provable data-independent accuracy guarantee. However, as a data-adaptive dimensionality reduction technique, when matrix $P$ is in fact low-rank (i.e., having good low-rank approximations), proximity embedding has the potential to achieve even better accuracy than proximity sketch. Our empirical results in Section 4 suggest that this is indeed the case for the Katz measure.

\subsubsection{Incremental Proximity Update}

To enable online proximity estimation, we periodically checkpoint $M$ and use the above dimensionality reduction techniques to approximate $P$ for the last checkpoint of $M$. Between two checkpoints, we apply an incremental update algorithm to approximate $P^{\prime}=\left(I-\beta \cdot M^{\prime}\right)^{-1}$, where $M^{\prime}=M+\Delta$ is the current matrix. Our algorithm is based on the second-order approximation of $P^{\prime}$ :

$$
\begin{aligned}
P^{\prime} & =[I-\beta(M+\Delta)]^{-1} \\
& \approx(I-\beta M)^{-1}+\beta \Delta+\beta^{2}\left(\Delta M+M \Delta+\Delta^{2}\right)
\end{aligned}
$$


The second-order approximation works well as long as $\Delta$ has only few non-zero elements and $\beta$ is small, making higher order terms negligible.

To estimate an individual element $P^{\prime}[x, y]$, we simply use:

$$
\begin{gathered}
P^{\prime}[x, y] \approx P[x, y]+\beta \Delta[x, y]+\sum_{k: \Delta[x, k] \neq 0} \beta^{2} \Delta[x, k] M[k, y]+ \\
\sum_{k: \Delta[k, y] \neq 0} \beta^{2} M[x, k] \Delta[k, y]+\sum_{k: \Delta[x, k] \neq 0} \beta^{2} \Delta[x, k] \Delta[k, y]
\end{gathered}
$$

If we checkpoint $M$ frequently enough, the difference between the last checkpoint $M$ and the current matrix $M^{\prime}$ will be quite small. In other words, the difference matrix $\Delta$ is likely to be sparse. As a result, we expect row $\Delta[x, *]$ and column $\Delta[*, y]$ to have few non-zero elements. By leveraging such sparseness, we can efficiently compute Eq. 22 in an online fashion. We demonstrate the efficiency and accuracy of our incremental proximity update algorithm in Section 4.2.3.

\section{LINK PREDICTION TECHNIQUES}

We use link prediction as a significant application of our proximity estimation methods. Our goal is to understand (i) the effectiveness of various proximity measures in the context of link prediction, and (ii) the benefit of combining multiple proximity measures. In this section, we summarize the link predictors and the proximity measures we use.

\subsection{Link Predictors}

We consider two types of link predictors: (i) basic link predictor that uses a single proximity measure, and (ii) composite link predictor that uses multiple proximity measures.

Basic link predictors. A basic link predictor consists of a proximity measure prox $[*, *]$, and a threshold $T$. Given an input graph $G=(V, E)$ (which models a past snapshot of a given social network), a node pair $\langle x, y\rangle \notin E$ is predicted to form an edge in the future if and only if the proximity between $x$ and $y$ is sufficiently large, i.e., $\operatorname{prox}[x, y] \geq T$.

Composite link predictors. A composite link predictor uses machine learning techniques to make link predictions based on multiple proximity measures. We use the WEKA machine learning package [21] to automatically generate composite link predictors using a number of machine learning algorithms, including the REPtree decision tree learner, J48 decision tree learner, JRip rule learner, support vector machine (SVM) learner, and Adaboost learner. The results are consistent across different learners we use. So we only report the results of the REPtree decision tree learner. REPtree is a variant of the commonly used C4.5 decision tree learning algorithm [46]. It builds a decision tree using information gain and prunes it using reduced error pruning. It allows direct control on the depth of the learned decision tree, making it easy to visualize and interpret the resulting composite link predictor.

\subsection{Proximity Measures}

We consider three classes of proximity measures summarized in Table 1, which are based on (i) graph distance, (ii) node neighborhood, and (iii) ensemble of paths, respectively.

Notations. We model a social network as a graph $G=(V, E)$, where $V$ is the set of nodes, and $E$ is the set of edges. $G$ can be either directed or undirected. For a node $x$, let $N(x)=\{y \mid\langle x, y\rangle \in$ $E\}$ be the set of neighbors $x$ has in $G$. Similarly, let $N^{-1}(x)=$ $\{y \mid\langle y, x\rangle \in E\}$ be the set of inverse neighbors $x$ has in $G$ (i.e., nodes that have $x$ as their neighbors). Let $A$ be the adjacency ma-

\begin{tabular}{|c|c|}
\hline graph distance & $\begin{array}{l}\mathrm{GD}[x, y]=\text { negated distance of the shortest } \\
\text { path from } x \text { to } y\end{array}$ \\
\hline common neighbors & $\mathrm{CN}|x, y|=|N(x) \cap N(y)|$ \\
\hline Adamic/Adar & $\mathrm{AA}[x, y]=\sum_{z \in N(x) \cap N(y) \frac{1}{\log \mid N(z)}}$ \\
\hline preferential attachment & $\overline{\mathrm{PA}}[x, y]=|N(x)| \cdot|N(y)|$ \\
\hline PageRank product & $\begin{array}{l}\mathrm{PRP}[x, y]=P R(x) \cdot P R(y), \text { where } \\
P R(x)=\frac{1-d}{V \mid}+d \sum_{z \in N^{-1}(x)} \frac{P R(z)}{N(x)}\end{array}$ \\
\hline Katz & $\begin{array}{l}\text { Katz }[x, y]=\sum_{\ell=1}^{\infty} \beta^{\ell} \cdot \mid \text { paths }_{x, y}^{\langle\ell\rangle} \mid \\
\text { we have: Katz }=(I-\beta A)^{-1}-I\end{array}$ \\
\hline
\end{tabular}
trix for $G$ (defined in Eq. 2). Let $T=D^{-1} A$ be the adjacency
Table 1: Summary of proximity measures

matrix with row sums normalized to 1 , where $D$ is a diagonal matrix with $D[i, i]=\sum_{j} A[i, j]$.

Graph distance based proximity measure. Perhaps the most direct metric for quantifying how close two nodes are is the graph distance. We thus define a proximity measure $\mathrm{GD}[x, y]$ as the negative of the shortest-path distance from $x$ to $y$. Note that the use of negated (instead of original) shortest-path distance ensures that the proximity measure $\mathrm{GD}[x, y]$ increases as $x$ and $y$ get closer.

Note that it is inefficient to apply Dijkstra's algorithm to compute shortest path distance from $x$ to $y$ when $G$ has millions of nodes. Instead, we exploit the small-world property [27] of the social network and apply expanded ring search to compute the shortest path distance from $x$ to $y$. Specifically, we initialize $\mathcal{S}=\{x\}$ and $\mathcal{D}=\{y\}$. In each step we either expand set $\mathcal{S}$ to include its members' neighbors (i.e., $\mathcal{S}=\mathcal{S} \cup\{v \mid\langle u, v\rangle \in E \wedge u \in \mathcal{S}\}$ ) or expand set $\mathcal{D}$ to include its members' inverse neighbors (i.e., $\mathcal{D}=\mathcal{D} \cup\{u \mid\langle u, v\rangle \in E \wedge v \in \mathcal{D}\})$. We stop whenever $\mathcal{S} \cap \mathcal{D} \neq \emptyset$ - the number of steps taken so far gives the shortest path distance. For efficiency, we always expand the smaller set between $\mathcal{S}$ and $\mathcal{D}$ in each step. We also stop when a maximum number of steps is reached (set to 6 in our evaluation).

Node neighborhood based proximity measures. We define four proximity measures based on node neighborhood.

- Common neighbors. For two nodes $x$ and $y$, they are more likely to become friends when the overlap of their neighborhoods is large. The simplest form of this approach is to count the size of the intersection: $\mathrm{CN}[x, y]=|N(x) \cap N(y)|$.

- Adamic/Adar. Like common neighbors, Adamic/Adar [1] also tries to measure the size of the intersection of two neighborhoods. However, Adamic/Adar also takes "rareness" into account, giving more weights to the common node with smaller number of friends: $\mathrm{AA}[x, y]=\sum_{z \in N(x) \cap N(y)} \frac{1}{\log |N(z)|}$.

- Preferential attachment. The preferential attachment is based on the idea that having a new neighbor is proportional to the size of the current neighborhood. Moreover, the probability of two users becoming friends is proportional to the product of the number of the current friends. We therefore define a proximity measure: $\mathrm{PA}[x, y]=|N(x)| \cdot|N(y)|$.

- PageRank product. PageRank is developed to analyze the hyperlink structure of Web pages by treating a hyperlink as a vote. The PageRank of a node depends on the count of inbound links and the PageRank of outbound neighbors. Formally, the PageRank of a node $x$, denoted as $P R(x)$, is defined recursively on $G=(V, E)$ as

$$
P R(x)=\frac{1-d}{|V|}+d \sum_{z \in N^{-1}(x)} \frac{P R(z)}{|N(x)|}
$$

where $d$ is a damping factor. We define the PageRank product of two nodes $x$ and $y$ as the product of two PageRank values: $\operatorname{PRP}[x, y]=P R(x) \cdot P R(y)$. 
Path-ensemble based proximity measures. We use the Katz measure $(\operatorname{Katz}[x, y])$ as a path-ensemble based proximity measure (described in Section 2.1). We use the Katz measure as the representative of path-ensemble based proximity measures for two main reasons. First, as shown in [30,31], the Katz measure is the more effective than other path-ensemble based proximity measures such as the rooted PageRank. Second, our results in Section 4 show that the accuracy of our proximity estimation methods is the highest for the Katz measure.

\section{EVALUATION}

\subsection{Dataset Description}

\begin{tabular}{|c|c|c|c|c|c|}
\hline & Snapshot & \# of Conn- & \# of & \# of Added & $\begin{array}{l}\text { Asymm } \\
\text { ink Fra }\end{array}$ \\
\hline Network & Dat & ected Nodes & Links & Links & Fr: \\
\hline \multirow[t]{3}{*}{ Digg } & $\overline{9 / 15 / 2008}$ & $\mid \overline{535,071}$ & $\overline{4,432,726}$ & & \multirow{3}{*}{$58.3 c^{\circ}$} \\
\hline & $10 / 25 / 2008$ & 567,771 & $4,813,668$ & 656,478 & \\
\hline & $11 / 10 / 2008$ & 567,771 & $4,941,401$ & 175,958 & \\
\hline \multirow[t]{3}{*}{ Flickr } & $3 / 01 / 2007$ & $1,932,735$ & $26,702,209$ & & \multirow{3}{*}{$37.8 \%$} \\
\hline & $4 / 15 / 2007$ & $2,172,692$ & $30,393,940$ & $3,691,731$ & \\
\hline & $5 / 18 / 2007$ & $2,172,692$ & $32,399,243$ & $2,005,303$ & \\
\hline Live- & $11 / 13 / 2008$ & $1,769,493$ & $61,488,262$ & & \multirow{3}{*}{$28.3 \%$} \\
\hline \multirow[t]{2}{*}{ Journal } & $12 / 05 / 2008$ & $1,769,543$ & $61,921,736$ & $1,566,059$ & \\
\hline & $1 / 30 / 2009$ & $1,769,543$ & $62,843,995$ & $3,093,064$ & \\
\hline \multirow[t]{3}{*}{ MySpace } & $12 / 11 / 2008$ & $2,128,945$ & $89,138,628$ & & \\
\hline & $1 / 11 / 2009$ & $2,137,773$ & $90,629,452$ & $1,845,898$ & \\
\hline & $2 / 14 / 2009$ & $2,137,773$ & $89,341,780$ & 696,016 & \\
\hline \multirow[t]{3}{*}{ YouTube } & $4 / 30 / 2007$ & $2,012,280$ & $9,762,825$ & & \multirow{3}{*}{0} \\
\hline & $6 / 15 / 2007$ & $2,532,050$ & $13,017,064$ & $3,254,239$ & \\
\hline & $7 / 23 / 2007$ & $2,532,050$ & $15,337,226$ & $2,320,162$ & \\
\hline \multirow{3}{*}{ Wikipedia } & $9 / 30 / 2006$ & $1,636,961$ & $28,950,137$ & & \multirow{3}{*}{83.1} \\
\hline & $12 / 31 / 2006$ & $1,758,323$ & $33,974,708$ & $5,024,571$ & \\
\hline & $4 / 06 / 2007$ & $1,758,323$ & $38,349,329$ & 4,37 & \\
\hline
\end{tabular}

Table 2: Dataset summary

We carry out our evaluation on five popular online social networks: Digg [14], Flickr [20], LiveJournal [33], MySpace [40], and YouTube [55]. For comparison, we also examine the hyperlink structure of Wikipedia [54]. For each network, we conduct three crawls and make three snapshots of the network. Table 2 summarizes the characteristics of the three snapshots for each of the networks. Note that, for the purpose of link prediction, we only use connected nodes (i.e., nodes with at least one incoming or outgoing friendship link), rather than considering all the crawled nodes. Another point to note is that since link prediction implies that based on one snapshot of the network, we predict the new links that are formed in the next snapshot, the same set of users should appear in two consecutive snapshots. Hence, for a growing network, the number of users appearing in the last snapshot that we create may be less than the total number of users (to match the previous snapshot). Lastly, although there can be both link additions and deletions between two snapshots, since the goal of link prediction is to predict those that get added, we explicitly show the number of added links between two consecutive snapshots in Table 2 .

Digg [14] is a website for users to share interesting Web content by posting a link to it. The posted link can be voted as either positive ("digg") or negative ("bury") by other users. Digg allows a user to become a "fan" of other users, which we consider as a friendship relation. All the friendship links together form a directed social graph. Overall, 58.3\% directly connected user pairs in Digg have asymmetric friendship (i.e., friendship link only exists in one direction between two users). We obtained the entire list of 1.9 million users in September 2008. We crawled friendship links among these users using the Digg API [15] in September 2008, October 2008, and November 2008. The resulting snapshots contain more than
500,000 connected users (i.e., users with at least one incoming or outgoing friendship link) out of 1.9 million crawled users.

Flickr [20] is a popular photo-sharing website. Flickr allows users to add other people as "contacts" to form a directed social link. We use the Flickr dataset collected by [36], which represents a breadth first search on the graph from a set of seed users. The dataset gives the growth of Flickr for 104 days and contains 33 million links among 2.3 million users. We treat the first 25 days as the bootstrap period to ensure that the crawl has sufficiently stabilized. We then partition the remaining dataset into three snapshots separated approximately by 40 days each. Note that, the third snapshot of Flickr contains links for the same 2.17 million users that appear in the second snapshot (and not the entire 2.3 million users).

LiveJournal [33] is a Web community that allows its users to post entries to personal journals. LiveJournal also acts as a social networking site, where a user can become a "fan" of another LiveJournal user. We consider this "fan" relationship as a directed friendship link in the social graph. Since LiveJournal does not provide a complete list of users, we obtained a list of active users who have published posts by analyzing periodic RSS announcements of recently updated journals starting from July 2008. We then used the LiveJournal API to gather friendship information of 2.2 million active users in November 2008, December 2008, and January 2009. The resulting snapshots have about 1.8 million connected users who have non-zero friendship links.

MySpace [40] is a social networking site where users can interact with each other by personalizing pages, commenting on others' photos and videos, and making friends. For two MySpace users to become friends, both parties have to agree. Therefore, the social links in MySpace are undirected and thus symmetric. We crawled 10 million MySpace users out of over 400 million users by taking the first 10 million user IDs in December 2008, January 2009, and February 2009. After discarding all the inactive, deleted, private, and solitary MySpace IDs, we get information for approximately 2.1 million users in each resulting snapshot.

YouTube [55] is a popular video-sharing website. Registered users can connect with others by creating friendship links. We use the undirected version of the social graph collected by [36], which covers the growth of YouTube for 165 days with 18 million added links among 3.2 million users. We divide the dataset into three snapshots separated by 45 days each. Note that the third snapshot of YouTube in Table 2 contains links for 2.5 million users that also appear in the second snapshot (and not the entire 3.2 million users).

Wikipedia [54] is an online encyclopedia which takes users' collaboration to build content. Different wiki pages are connected through hyperlinks. We compare Wikipedia's hyperlink structure against social graphs of users from the previous five online social networks. Similar to general Web pages, most links in Wikipedia are asymmetric. We use the data collected by [36] over a six-year period from 2001 to 2007, which contains 38 million links connecting 1.8 million pages. We extract three snapshots separated approximately by 90 days each.

\subsection{Proximity Estimation Algorithms}

In this section, we evaluate the accuracy and scalability of our proximity estimation methods using the above six datasets. We present results for Katz and RPR (defined in Section 2). The accuracy for escape probability (EP) is similar to RPR (due to their close relationship in Eq. 5) and is omitted in the interest of brevity.

Accuracy metrics. We quantify the estimation error using three different metrics: (i) Normalized Absolute Error (NAE) (defined as $\frac{\mid e^{2} t_{i}-\text { actual }_{i} \mid}{\operatorname{mean}_{i}\left(\text { actual }_{i}\right)}$ ), (ii) Normalized Mean Absolute Error (NMAE) (defined as $\frac{\sum_{i} \mid \text { est }_{i}-\text { actual }_{i} \mid}{\sum_{i} \text { actual }_{i}}$ ), and (iii) Relative Error (defined as 


\begin{tabular}{|l|r|r|}
\hline Network & PageRank based selection & Uniform selection \\
\hline \hline Digg & 0.00015 & 0.00023 \\
\hline Flickr & 0.00010 & 0.00238 \\
\hline LiveJournal & 0.01222 & 0.07322 \\
\hline MySpace & 0.00016 & 0.00032 \\
\hline YouTube & 0.02115 & 0.05410 \\
\hline Wikipedia & 0.00266 & 0.00328 \\
\hline
\end{tabular}

Table 3: NMAE of different landmark selection schemes.

$\left.\frac{\mid e^{2} t_{i}-\text { actual }_{i} \mid}{\text { actual }_{i}}\right)$, where $e t_{i}$ and actual $_{i}$ denote the estimated and actual values of the proximity measure for node pair $i$, respectively.

Since it is expensive to compute the actual proximity measures over all the data points, we randomly sample 100,000 data points by first randomly selecting 200 rows from the proximity matrix and then selecting 500 elements from each of these rows. We then compute errors for these 100,000 data points.

\subsubsection{Proximity Embedding}

We first evaluate the accuracy of proximity embedding. We aim to answer the following questions: (i) How accurate is proximity embedding in estimating Katz and RPR? (ii) How many dimensions and landmarks are required to achieve high accuracy? (iii) How does the landmark selection algorithm affect accuracy?

Parameter settings. Throughout our evaluation, we use a damping factor of $\beta=0.05, \ell_{\max }=6$, and 1600 landmarks unless otherwise specified. We also vary these parameters to understand their impact. By default, we select landmarks based on the PageRank of each node. Specifically, we first compute PageRank for each node and normalize the sum of PageRank of all nodes to 1 . We then use the normalized PageRank as the probability of assigning a node as a landmark. In this way, nodes with high PageRank values are more likely to become landmarks. For comparison, we also examine the performance of uniform landmark selection, which selects landmarks uniformly at random.

Varying the number of dimensions. Figure 3 plots the CDF of normalized absolute errors in approximating the Katz measure as we vary the number of dimensions from 5 to 60 . We make the following two key observations. First, for all six datasets the normalized absolute error is small: in more than $95 \%$ cases the normalized absolute error is within 0.05 and NMAE is within 0.05 except YouTube. The error in YouTube is higher because its "intrinsic" dimensionality is higher as analyzed in Figure 6 (see below). Second, as we would expect, the error decreases with the number of dimensions. The reduction is more significant in the YouTube dataset, because the other datasets have very low "intrinsic" dimensionality and using only 5 dimensions already gives low approximation error, whereas YouTube has higher "intrinsic" dimensionality and increasing the number of dimensions is more helpful.

Relative errors. Figure 4 further plots the CDF of relative errors using 60 dimensions. We take top $1 \%, 5 \%$, and $10 \%$ of the randomly selected data points and generate the CDF for each of the selections. In all datasets, we observe that the relative errors are smaller for elements with larger values. This is desirable because larger elements play a more important role in many applications and are thus more important to estimate accurately.

Uniform landmark selection. Table 3 compares the NMAE of PageRank based landmark selection and uniform selection. PageRank based selection yields higher accuracy than uniform selection. It reduces NMAE by 35\% for Digg, $95.8 \%$ for Flickr, $83.3 \%$ for LiveJournal, $50 \%$ for MySpace, $61 \%$ for YouTube, and $19 \%$ for Wikipedia. The reason is that high-PageRank nodes are well connected, and it is less likely for nodes to be far away from all such landmarks, thereby improving the estimation accuracy.

\begin{tabular}{|c|c|c|c|}
\hline \multirow[b]{2}{*}{ Network } & \multicolumn{3}{|c|}{ Threshold for Large Katz Values } \\
\hline & $1 \%$ row sum & $0.1 \%$ row sum & $0 \%$ row sum \\
\hline$\overline{\overline{\text { Digg }}}$ & $\overline{0.0562}$ & $\overline{0.0650}$ & $\overline{0.0002}$ \\
\hline Flickr & 0.2177 & 0.2505 & 0.0001 \\
\hline LiveJournal & 0.9872 & 0.2516 & 0.0122 \\
\hline MySpace & 0.0532 & 0.0650 & 0.0002 \\
\hline YouTube & 0.0074 & 0.0054 & 0.0212 \\
\hline Wikipedia & 0.0039 & 0.0001 & 0.0027 \\
\hline
\end{tabular}

(a) NMAE of proximity embedding

\begin{tabular}{|l|r|r|r|}
\hline \multirow{2}{*}{ Network } & \multicolumn{3}{|c|}{ Threshold for Large Katz Values } \\
\cline { 2 - 4 } & 1\% row sum & 0.1\% row sum & 0\% row sum \\
\hline \hline Digg & 0.0001 & 0.3209 & 211.5 \\
\hline Flickr & 0.0048 & 0.0293 & 1116.3 \\
\hline LiveJournal & 0.0012 & 0.0113 & 1383.2 \\
\hline MySpace & 0.0041 & 0.0360 & 1451.1 \\
\hline YouTube & 0.0495 & 0.3769 & 1141.3 \\
\hline Wikipedia & 0.0114 & 0.2645 & 647.3 \\
\hline
\end{tabular}

(b) NMAE of proximity sketch

Table 4: Comparing proximity embedding and proximity sketch in estimating large Katz values.

\begin{tabular}{|l|r|r|r|}
\hline \multirow{2}{*}{ Network } & \multicolumn{3}{|c|}{ Threshold for Large RPR Values } \\
\cline { 2 - 4 } & 1\% row sum & 0.1\% row sum & 0\% row sum \\
\hline \hline Digg & 0.6662 & 2.0008 & 0.7933 \\
\hline Flickr & 0.7285 & 1.6385 & 1.0000 \\
\hline LiveJournal & 1.4491 & 7.2752 & 0.9980 \\
\hline MySpace & 1.0916 & 6.6324 & 0.9984 \\
\hline YouTube & 0.7068 & 1.1952 & 1.0635 \\
\hline Wikipedia & 1.4429 & 5.7987 & 0.7208 \\
\hline
\end{tabular}

(a) NMAE of proximity embedding

\begin{tabular}{|l|r|r|r|}
\hline \multirow{2}{*}{ Network } & \multicolumn{3}{|c|}{ Threshold for Large RPR Values } \\
\cline { 2 - 4 } & 1\% row sum & 0.1\% row sum & 0\% row sum \\
\hline \hline Digg & 0.0031 & 0.0247 & 131.8 \\
\hline Flickr & 0.0006 & 0.0019 & 717.6 \\
\hline LiveJournal & 0.0042 & 0.0296 & 500.2 \\
\hline MySpace & 0.0038 & 0.0269 & 853.0 \\
\hline YouTube & 0.0019 & 0.0110 & 486.3 \\
\hline Wikipedia & 0.0046 & 0.0265 & 619.7 \\
\hline
\end{tabular}

(b) NMAE of proximity sketch

Table 5: Comparing proximity embedding and proximity sketch in estimating large RPR values.

Varying the number of landmarks. Figure 5 shows the NMAE as we vary the number of landmarks. As before, we use PageRank based landmark selection. For all the datasets that we use, NMAE values decrease with the number of landmarks. The decrease is sharp when the number of landmarks is small, and then tapers off as the number of landmarks reaches 100-200. In all cases, 1600 landmarks are large enough and further increasing the value yields only marginal benefit if any.

Estimating large Katz values. Table 4(a) shows the accuracy of proximity embedding in estimating Katz values larger than $1 \%$, $0.1 \%$ and $0 \%$ of their corresponding row sums in the Katz matrix. As we can see, for elements larger than 0, the NMAE is low (the largest one is 0.0212 for YouTube). In comparison, for elements larger than $1 \%$ and $0.1 \%$ of the row sums, the NMAE is often larger (e.g., the corresponding values for LiveJournal are 0.98 and 0.25 ). Manual inspection suggests that many Katz values larger than $1 \%$ of row sum involve a direct link between two nodes in an isolated island of the network that cannot reach any landmarks. For such node pairs, the proximity embedding yields an estimate of 0 , thus seriously inflating the NMAE. Fortunately, large Katz values are quite rare in each row. As a result, the NMAE is low when we consider all elements in the Katz matrix.

Estimating large Rooted PageRank values. Table 5(a) shows the accuracy of proximity embedding in estimating Rooted PageRank 


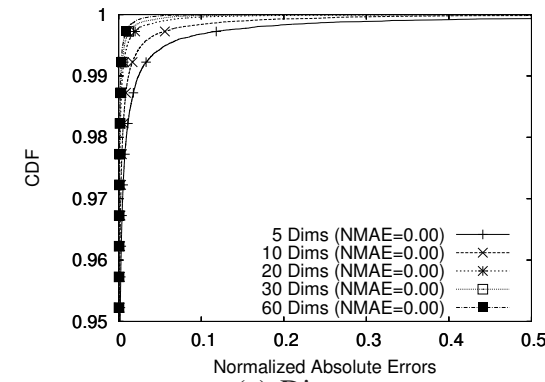

(a) Digg

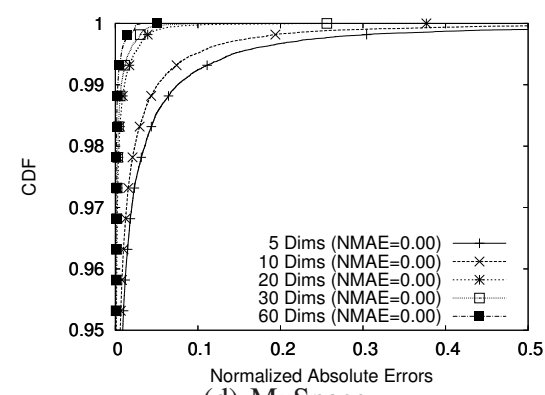

(d) MySpace

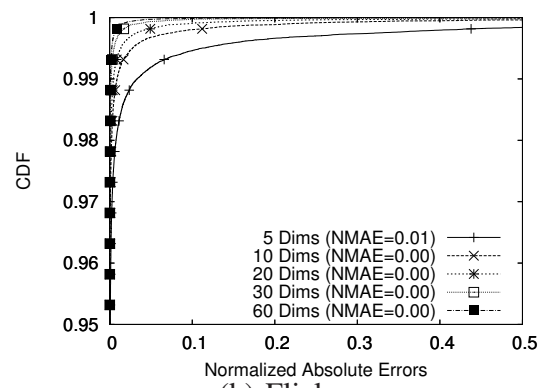

(b) Flickr

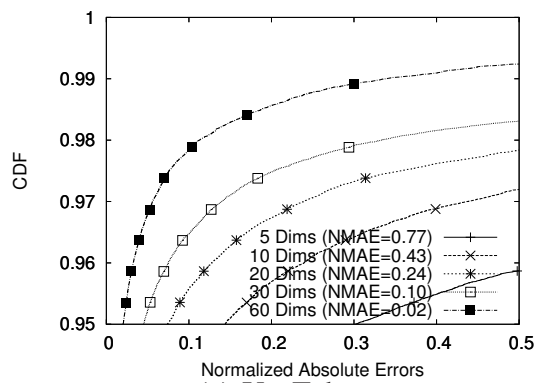

(e) YouTube

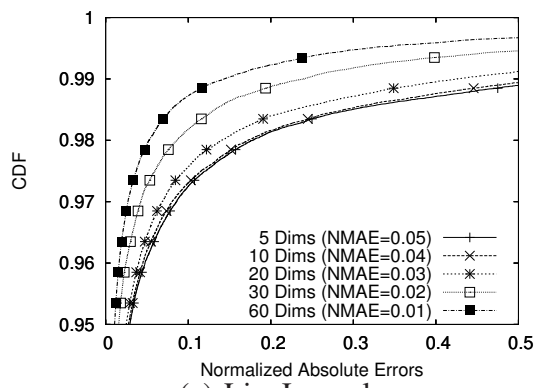

(c) LiveJournal

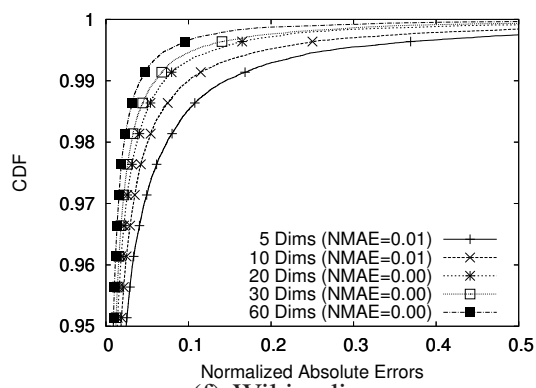

(f) Wikipedia

Figure 3: Normalized absolute errors with a varying number of dimensions (Katz measure, $\beta=0.05$, and 1600 landmarks).

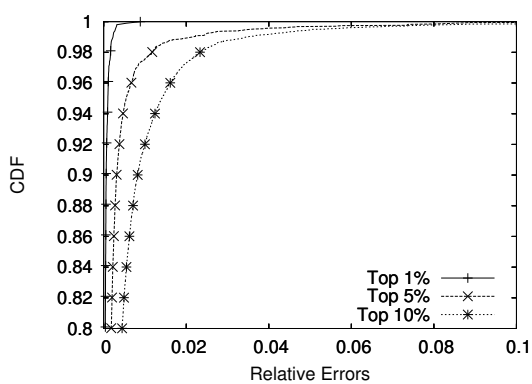

(a) Digg

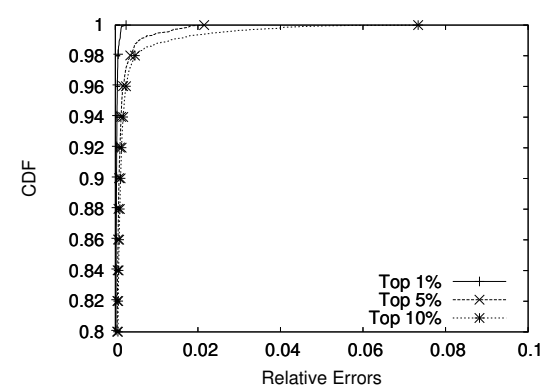

(d) MySpace

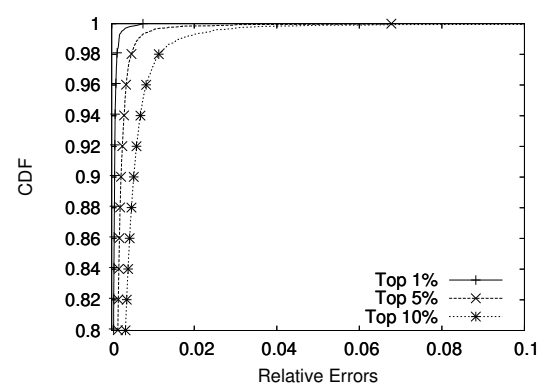

(b) Flickr

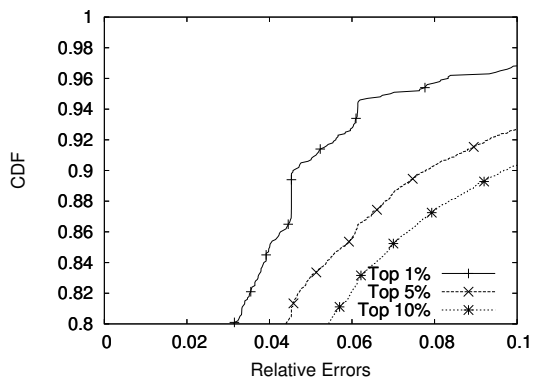

(e) YouTube

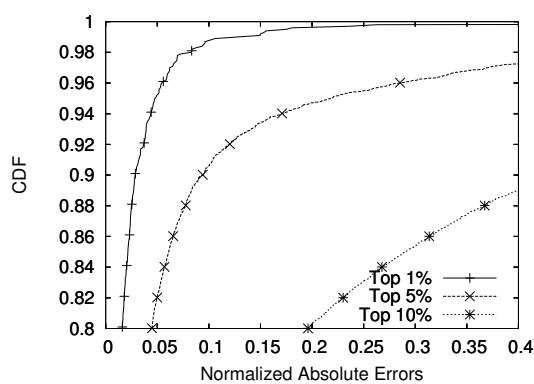

(c) LiveJournal

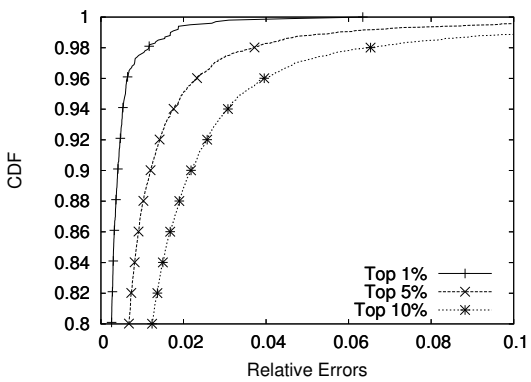

(f) Wikipedia

Figure 4: Relative errors for top 1\%, $5 \%$, and $10 \%$ largest values (Katz measure, $\beta=0.05$, 1600 landmarks, and 60 dimensions).

values larger than $1 \%, 0.1 \%$, and $0 \%$ of their corresponding row sums in the RPR matrix. We observe that the NMAE for RPR is much larger than the NMAE for Katz.

To understand why proximity embedding performs well on Katz but not on RPR, we plot the fraction of total variance captured by the best rank- $k$ approximation to the inter-landmark proximity matrices Katz $[L, L]$ and $\operatorname{RPR}[L, L]$ in Figure 6, where $L$ is the set of landmarks. Note that the best rank- $k$ approximation to a matrix can be easily computed through the use of singular value decomposition (SVD). The smaller the number of dimensions (i.e., $k$ ) it takes to capture most variance of the matrix, the lower the "intrinsic" dimensionality the matrix has. As we can see, for LiveJournal, even
3 dimensions can capture over 99\% variance for Katz, whereas it takes 1590 dimensions to achieve similar approximation accuracy for rooted PageRank. This indicates that the RPR matrix is not lowrank, whereas the Katz matrix exhibits low-rank property, which makes proximity embedding work well.

\subsubsection{Proximity Sketch}

Now we evaluate the accuracy of proximity sketch. We use $H=$ 3 hash tables and $c=1600$ columns in each table.

Estimating large Katz values. Table 4(b) shows the NMAE of proximity sketch in estimating Katz values larger than $1 \%, 0.1 \%$, and $0 \%$ of row sum. Comparing Table 4(a) and (b), we observe 


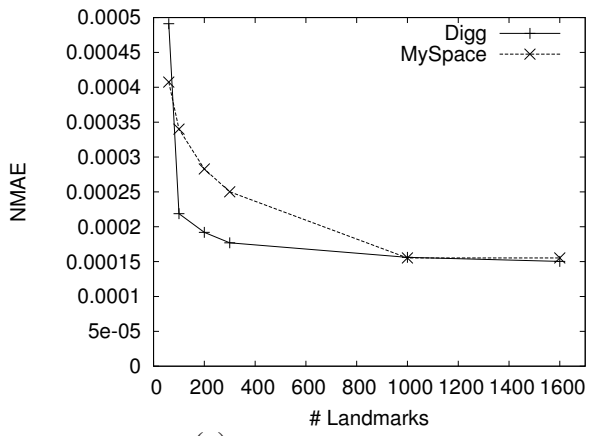

(a) Digg and MySpace

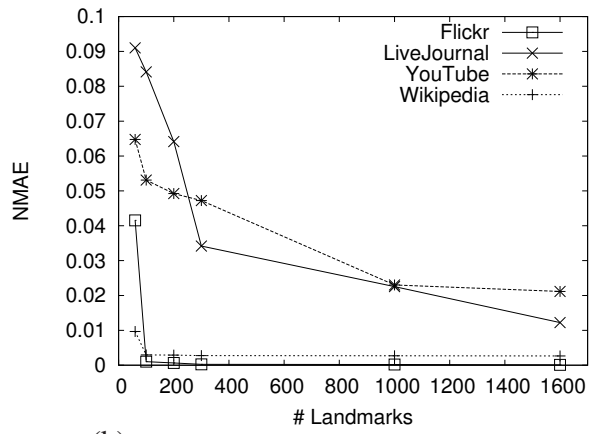

(b) Flickr, LiveJournal, YouTube, Wikipedia

Figure 5: NMAE comparison of different number of landmarks under PageRank based landmark selection.

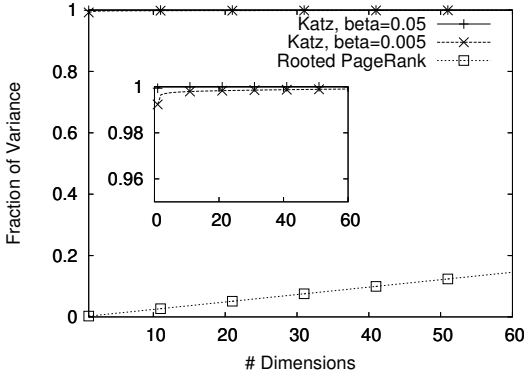

(a) Digg

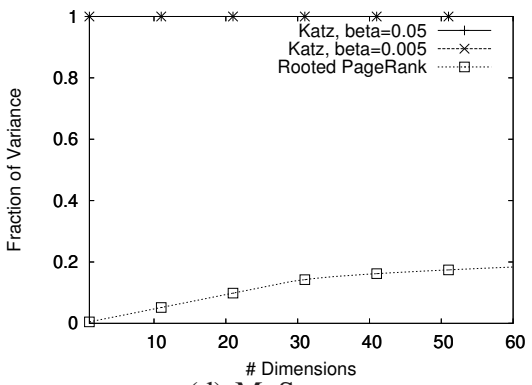

(d) MySpace

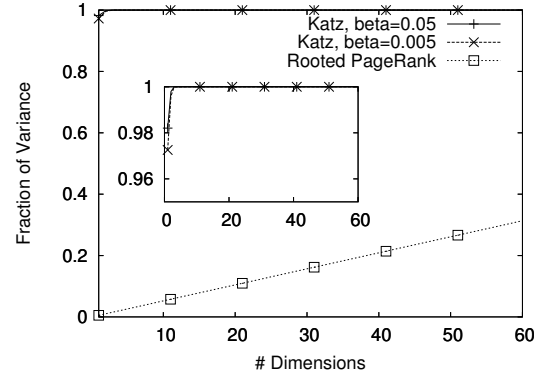

(b) Flickr

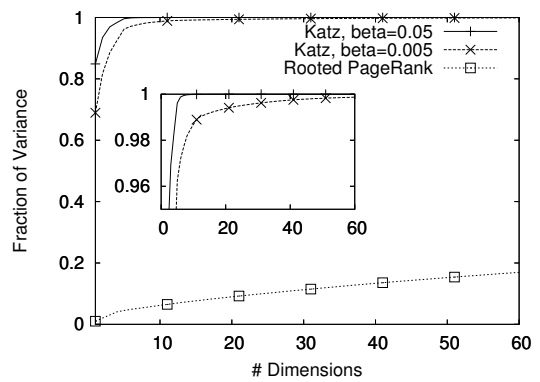

(e) YouTube

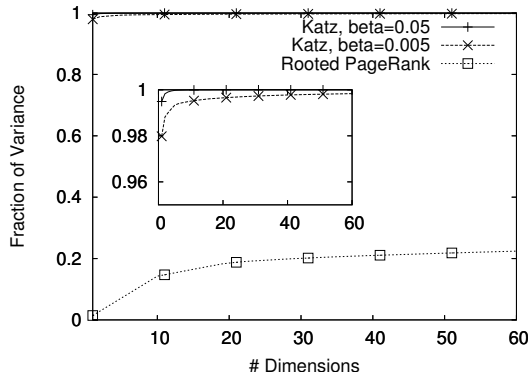

(c) LiveJournal

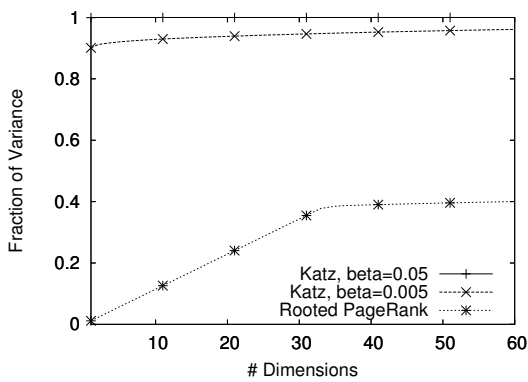

(f) Wikipedia

Figure 6: Fraction of total variance captured by the best rank- $k$ approximation to inter-landmark proximity matrices $\mathrm{Katz}[L, L]$ and $\operatorname{RPR}[L, L]$.

that proximity sketch generally performs better than proximity embedding for large values (i.e., greater than $1 \%$ of row sums), and performs worse for small values (i.e., greater than $0.1 \%$ and 0 of row sums). This is consistent with our expectation, since proximity sketch is designed to approximate large elements. This also suggests that the two algorithms are complimentary and we can potentially have a hybrid algorithm that chooses the results among the two algorithms based on the magnitude of the estimated values.

Estimating large Rooted PageRank values. Table 5(b) shows the NMAE of proximity sketch in estimating RPR. As for Katz, proximity sketch yields lower error than proximity embedding for large elements (i.e., larger than $1 \%$, and $0.1 \%$ of row sums) and higher error for small elements (i.e., larger than 0). This confirms that proximity sketch is effective in estimating large elements.

\subsubsection{Incremental Proximity Update}

Next, we evaluate the accuracy of our incremental proximity update algorithm. We use two checkpoints of crawl data that differ by one day: May 17-18, 2007 for Flickr, July 22-23, 2007 for YouTube, and April 5-6, 2007 for Wikipedia. The one-day gap between two checkpoints yields $0.3 \%, 0.5 \%$, and $0.05 \%$ difference between $M$ and $M^{\prime}$ for Flickr, YouTube, and Wikipedia, respec- tively. We do not use LiveJournal, MySpace, and Digg, since we have no checkpoints that are one day apart for these sites.

Figure 7 plots the CDF of normalized absolute errors and relative errors for the Katz measure using the incremental update algorithm in conjunction with proximity embedding (denoted as "Embed + inc. updates"). For comparison, we also plot the errors of using proximity embedding alone (denoted as "Prox. embedding only"). As we can see, the curves corresponding to incremental update are very close to those of proximity embedding. This indicates that we can use the incremental algorithm to efficiently and accurately update the proximity matrix as $M$ changes dynamically and only re-compute the proximity matrix periodically over multiple days.

\subsubsection{Scalability}

Table 6 shows the computation time for proximity embedding, proximity sketch, incremental proximity embedding, common neighbor, and shortest path computation. The measurements are taken on an Intel Core 2 Duo $2.33 \mathrm{GHz}$ machine with 4GB memory running Ubuntu Linux kernel v2.6.24. We explicitly distinguish the query time for positive and negative samples - A node pair $\langle A, B\rangle$ is considered a positive sample if there is a friendship link from $A$ to $B$; otherwise it is considered a negative sample. 


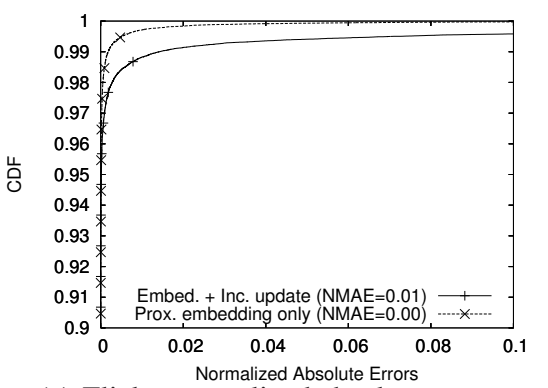

(a) Flickr, normalized absolute errors

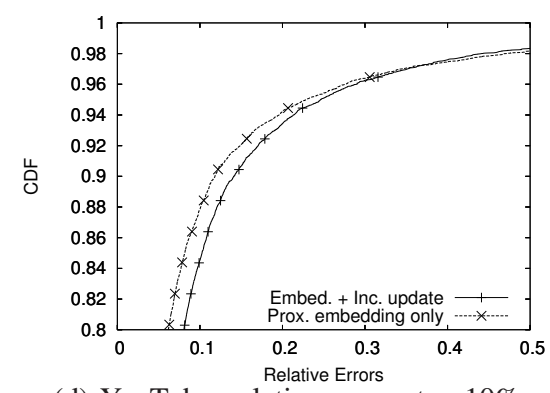

(d) YouTube, relative errors, top $10 \%$

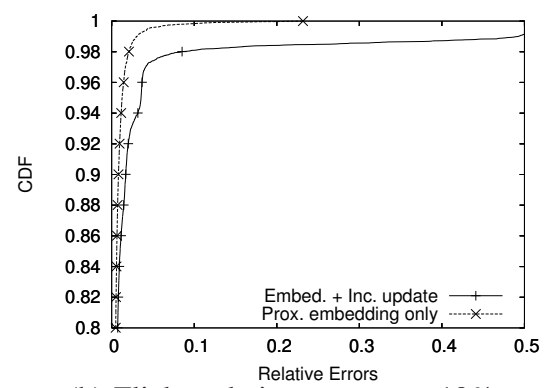

(b) Flickr, relative errors, top $10 \%$

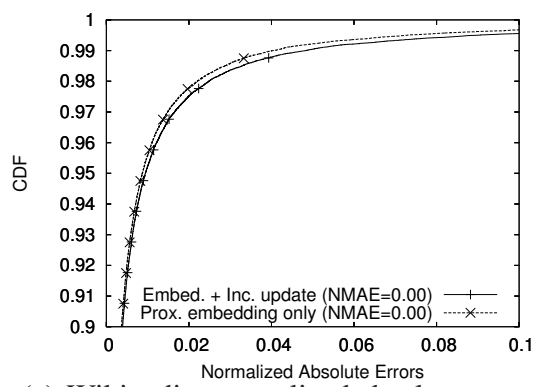

(e) Wikipedia, normalized absolute error

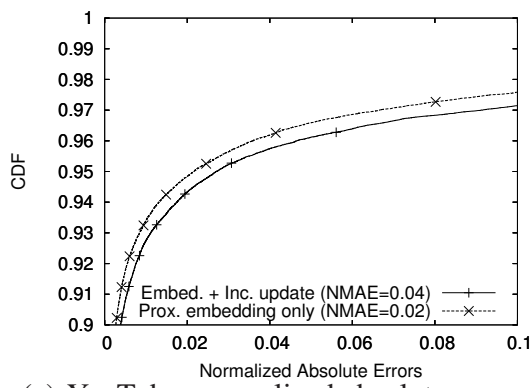

(c) YouTube, normalized absolute errors

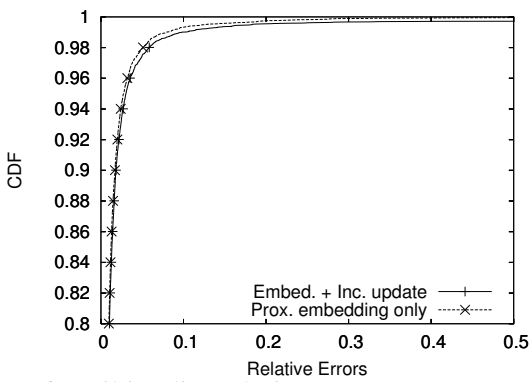

(f) Wikipedia, relative errors, top $10 \%$

Figure 7: Normalized absolute errors and relative errors of incremental update algorithm, Katz measure.

We make several observations. First, the query time for both proximity embedding and proximity sketch is small, even smaller than common neighbor and shortest path computation, which are traditionally considered much cheaper operations than computing Katz measure. Second, the preprocessing time of proximity sketch and proximity embedding increases linearly with the number of links in the dataset. As preprocessing can be done in parallel, we use the Condor system [17] for datasets with a large number of links. Running preprocessing step simultaneously from 150 machines, we observe that the total preprocessing time goes down to less than 30 minutes, even for large networks such as MySpace and LiveJournal. Furthermore, the pre-processing only needs to be done periodically (e.g., once every few days). For symmetric networks, such as MySpace and YouTube, proximity embedding only needs to compute either $P[L, *]$ or $P[*, L]$, reducing the preparation time to half of that of proximity sketch. Finally, the incremental proximity update algorithm eliminates pre-computation time at the cost of increased query time. However, even the increased query time is much smaller than shortest path computation. These results demonstrate the scalability of our approaches.

\subsubsection{Summary}

To summarize, our evaluation shows that proximity embedding and proximity sketch are complementary: the former is more accurate in estimating random samples, whereas the latter is more effective in estimating large elements. Comparing Katz and RPR, proximity embedding yields much more accurate estimation for Katz than for RPR due to the low-rank property in the Katz matrix. In particular, proximity embedding yields highly accurate Katz estimation for random samples (achieving NMAE of within 0.02).

Note that for the purpose of link prediction, it is insufficient to only estimate few large Katz values because (i) more than $15 \%$ of node pairs having Katz measure greater than $0.1 \%$ of row sums already have friendship (i.e., links) and (ii) for the remaining $85 \%$ node pairs, which are not already friends, the probability of nodepairs with the large Katz values becoming friends is only $0.21 \%$, whereas the average probability of random node-pairs becoming friends is $10.57 \%$. So we use proximity embedding for estimating Katz for link prediction in Section 4.3.

\subsection{Link Prediction Evaluation}

\subsubsection{Evaluation Methodology}

In the friendship link prediction problem, we construct the positive set as the set of user pairs that were not friends in the first snapshot, but become friends in the second snapshot. The negative set consists of user pairs that are friends in neither snapshots.

Metrics. We measure link prediction accuracy in terms of false negative rate $(F N)$, and false positive rate $(F P)$ :

$$
\begin{aligned}
F N & =\frac{\# \text { of missed friend links }}{\# \text { of new-friend links }} \\
F P & =\frac{\# \text { of incorrectly predicted friend links }}{\text { \#of non-friend links }}
\end{aligned}
$$

We also observe that not all proximity measures are applicable to all node pairs. For example, common neighbor is applicable only when nodes are two hops away. Thus we introduce another metric, called applicable ratio $(A R)$, which quantifies the fraction of node pairs for which a given proximity measure is non-zero.

Training and testing datasets. As shown earlier, we create three snapshots of friendship networks ( $S 1, S 2$, and $S 3$ ) for each network in Table 2 . We train link predictors by analyzing the differences in link relations of between $S 1$ and $S 2$, and predict who will likely make friendship relations in $S 3$. Therefore, the training set is based on the period from $S 1$ to $S 2$, and the testing set is based on the period from $S 2$ to $S 3$. Note that we only consider the common users of the two snapshots when we count the new friendship links. Since friendship relations in our datasets are very sparse, we sample about 50,000 positives and 200,000 negatives for both training and testing purposes.

Link predictors. We evaluate basic link predictors based on the following three classes of proximity measures.

- Distance based predictor: graph distance (GD).

- Node neighborhood based predictors: common neighbors (CN), Adamic/Adar (AA), preferential attachment (PA), and PageRank product (PRP).

- Path-ensemble based predictor: Katz (Katz). 


\begin{tabular}{|c|c|c|c|c|c|c|c|c|c|c|c|c|}
\hline Dataset & \multicolumn{3}{|c|}{ proximity embedding } & \multicolumn{3}{|c|}{ proximity sketch } & \multirow{2}{*}{\multicolumn{2}{|c|}{$\begin{array}{c}\text { Incremental update } \\
\text { Query }\end{array}$}} & \multirow{2}{*}{\multicolumn{2}{|c|}{$\frac{\text { Common neighbor }}{\text { Query }}$}} & \multirow{2}{*}{\multicolumn{2}{|c|}{$\begin{array}{c}\text { Shortest path distance } \\
\text { Query }\end{array}$}} \\
\hline Job type & Preprocess & $\mathrm{Qu}$ & ery & Preprocess & $\mathrm{Qu}$ & ery & & & & & & \\
\hline Sample type & & Pos & $\mathrm{Neg}$ & & Pos & Neg & Pos & Neg & Pos & Neg & Pos & $\mathrm{Neg}$ \\
\hline$\overline{\overline{\text { Digg }}}$ & $1.08 \mathrm{hrs}$ & $0.1 \mu \mathrm{s}$ & $0.1 \mu \mathrm{s}$ & $\overline{1.16 \mathrm{hrs}}$ & $0.3 \mu \mathrm{s}$ & $0.2 \mu \mathrm{s}$ & $\overline{70.3 \mu \mathrm{s}}$ & $\overline{21.1 \mu \mathrm{s}}$ & $\overline{15.0 \mu \mathrm{s}}$ & $\overline{12.0 \mu \mathrm{s}}$ & $\overline{149.2 \mu \mathrm{s}}$ & $\overline{132.5 \mu \mathrm{s}}$ \\
\hline Flickr & $4.26 \mathrm{hrs}$ & $47.9 \mu \mathrm{s}$ & $11.8 \mu \mathrm{s}$ & $4.88 \mathrm{hrs}$ & $45.2 \mu \mathrm{s}$ & $32.5 \mu \mathrm{s}$ & $1061.4 \mu \mathrm{s}$ & $457.7 \mu \mathrm{s}$ & $478.9 \mu \mathrm{s}$ & $118.0 \mu \mathrm{s}$ & $3111.1 \mu \mathrm{s}$ & $1720.4 \mu \mathrm{s}$ \\
\hline LiveJournal & $8.29 \mathrm{hrs}$ & $14.6 \mu \mathrm{s}$ & $13.7 \mu \mathrm{s}$ & $8.71 \mathrm{hrs}$ & $15.1 \mu \mathrm{s}$ & $13.4 \mu \mathrm{s}$ & $2528.7 \mu \mathrm{s}$ & $734.1 \mu \mathrm{s}$ & $546.2 \mu \mathrm{s}$ & $137.5 \mu \mathrm{s}$ & $9976.8 \mu \mathrm{s}$ & $2416.7 \mu \mathrm{s}$ \\
\hline MySpace & $4.92 \mathrm{hrs}$ & $58.8 \mu \mathrm{s}$ & $84.1 \mu \mathrm{s}$ & $9.85 \mathrm{hrs}$ & $62.0 \mu \mathrm{s}$ & $88.5 \mu \mathrm{s}$ & $6923.7 \mu \mathrm{s}$ & $1605.5 \mu \mathrm{s}$ & $1588.1 \mu \mathrm{s}$ & $841.8 \mu \mathrm{s}$ & $50273.3 \mu \mathrm{s}$ & $41473.2 \mu \mathrm{s}$ \\
\hline YouTube & $2.27 \mathrm{hrs}$ & $25.1 \mu \mathrm{s}$ & $20.6 \mu \mathrm{s}$ & $4.67 \mathrm{hrs}$ & $32.4 \mu \mathrm{s}$ & $35.6 \mu \mathrm{s}$ & $1681.5 \mu \mathrm{s}$ & $596.8 \mu \mathrm{s}$ & $251.6 \mu \mathrm{s}$ & $206.4 \mu \mathrm{s}$ & $3727.5 \mu \mathrm{s}$ & $1029.9 \mu \mathrm{s}$ \\
\hline Wikipedia & 3.30hrs & $0.5 \mu \mathrm{s}$ & $0.2 \mu \mathrm{s}$ & $3.75 \mathrm{hrs}$ & $0.6 \mu \mathrm{s}$ & $0.2 \mu \mathrm{s}$ & $104.0 \mu \mathrm{s}$ & $46.5 \mu \mathrm{s}$ & $54.0 \mu \mathrm{s}$ & $24.0 \mu \mathrm{s}$ & $1377.9 \mu \mathrm{s}$ & $374.6 \mu \mathrm{s}$ \\
\hline
\end{tabular}

Table 6: Computation time of proximity sketch, proximity embedding, common neighbor and shortest path distance.

\begin{tabular}{|l|c|r|r|r|r|r|r|}
\hline & & PA & PRP & CN & AA & Katz & GD \\
\hline \hline \multirow{3}{*}{ Digg } & positive & $90.8 \%$ & $100 \%$ & $32.4 \%$ & $32.1 \%$ & $56.6 \%$ & $46.5 \%$ \\
& negative & $2.7 \%$ & $100 \%$ & $1.2 \%$ & $1.1 \%$ & $29.9 \%$ & $35.6 \%$ \\
& all & $4.8 \%$ & $100 \%$ & $11.2 \%$ & $11.1 \%$ & $37.8 \%$ & $39.2 \%$ \\
\hline \multirow{3}{*}{ Flickr } & positive & $100 \%$ & $67.5 \%$ & $63.3 \%$ & $63.1 \%$ & $97.8 \%$ & $97.3 \%$ \\
& negative & $100 \%$ & $80.1 \%$ & $0.1 \%$ & $0.1 \%$ & $96.0 \%$ & $69.9 \%$ \\
& all & $100 \%$ & $77.5 \%$ & $12.8 \%$ & $12.8 \%$ & $96.4 \%$ & $75.4 \%$ \\
\hline \multirow{3}{*}{ LiveJournal } & positive & $99.4 \%$ & $100 \%$ & $27.5 \%$ & $27.5 \%$ & $74.7 \%$ & $98.6 \%$ \\
& negative & $93.1 \%$ & $100 \%$ & $0.2 \%$ & $0.03 \%$ & $91.8 \%$ & $93.1 \%$ \\
& all & $94.5 \%$ & $100 \%$ & $5.7 \%$ & $5.6 \%$ & $88.3 \%$ & $94.5 \%$ \\
\hline \multirow{3}{*}{ MySpace } & positive & $100 \%$ & $100 \%$ & $72.8 \%$ & $72.8 \%$ & $100 \%$ & $100 \%$ \\
& negative & $100 \%$ & $100 \%$ & $1.5 \%$ & $1.5 \%$ & $100 \%$ & $95.0 \%$ \\
& all & $100 \%$ & $100 \%$ & $15.3 \%$ & $15.3 \%$ & $100 \%$ & $95.9 \%$ \\
\hline \multirow{3}{*}{ YouTube } & positive & $100 \%$ & $100 \%$ & $31.8 \%$ & $31.9 \%$ & $99.1 \%$ & $100 \%$ \\
& negative & $100 \%$ & $100 \%$ & $0.3 \%$ & $0.2 \%$ & $91.2 \%$ & $100 \%$ \\
& all & $100 \%$ & $100 \%$ & $7.3 \%$ & $7.1 \%$ & $93.6 \%$ & $100 \%$ \\
\hline \multirow{3}{*}{ Wikipedia } & positive & $73.2 \%$ & $100 \%$ & $44.7 \%$ & $44.6 \%$ & $74.5 \%$ & $74.4 \%$ \\
& negative & $88.9 \%$ & $100 \%$ & $4.3 \%$ & $4.3 \%$ & $82.9 \%$ & $80.7 \%$ \\
& all & $85.1 \%$ & $100 \%$ & $14.3 \%$ & $14.3 \%$ & $80.9 \%$ & $79.1 \%$ \\
\hline
\end{tabular}

\begin{tabular}{|l|r|r|r|}
\hline Network & \# of high deg. links & \# of total links & \% of high deg. links \\
\hline \hline Digg & $1,860,703$ & $4,813,668$ & $38.6 \%$ \\
\hline Flickr & $23,535,630$ & $30,393,940$ & $77.4 \%$ \\
\hline LiveJournal & $60,058,439$ & $61,921,736$ & $96.9 \%$ \\
\hline MySpace & $43,877,840$ & $90,629,452$ & $48.4 \%$ \\
\hline YouTube & $9,392,367$ & $13,017,064$ & $72.1 \%$ \\
\hline Wikipedia & $18,936,865$ & $33,974,708$ & $55.7 \%$ \\
\hline
\end{tabular}

Table 8: Proportion of links connected to top $0.1 \%$ highest degree nodes in each network.

trade-off curve. In Wikipedia dataset, Katz performs slightly worse than PRP, which is optimized for predicting hyperlink structures. We will further investigate the reason behind the performance of Katz later in this section.

- Graph distance based proximity measure. The graph distance (denoted as GD) achieves high accuracy in Digg, LiveJournal, MySpace, Flickr and Wikipedia. But it has several important limitations. First, shortest path distance is determined solely by the shortest path and takes only integer values. This means that it has coarse granularity and introduces many ties. Second, the computation of shortest path distance is much more expensive in large networks [51] (also shown in Table 6).

- Effect of link symmetry. To understand the effect of link asymmetry, we make the friendship relation as reciprocal by adding reverse link in all existing node pairs. Table 2 shows the fraction of asymmetric links in our dataset. Figure 9 shows how the symmetric predictors performs in asymmetric datasets. In Wikipedia and Digg datasets, adding reverse edges improves the accuracy. But adding reverse edges does not always help; it stays almost the same in Flickr or a bit worse in LiveJournal.

A good empirical performance indicator. Despite the significant variation, we find that the ranking between sophisticated predictors such as Katz versus simple predictors such as common neighbors and Adamic/Adar can be qualitatively predicted based on the fraction of edges contributed by the highest degree nodes as follows.

Table 8 shows the number of links incoming and outgoing from the highest degree nodes, the number of links in the entire network and their proportion, where a node is considered a high degree node if its node degree (combining incoming and outgoing degrees) is among the top $0.1 \%$ highest node degrees. Ranking different networks based on the percentage of links connected to such highest degree nodes (in decreasing order), we obtain:

$$
\begin{aligned}
\text { LiveJournal } & >\text { Flickr }>\text { YouTube } \\
& \gg \text { Wikipedia }>\text { MySpace }>\text { Digg }
\end{aligned}
$$

- Path-ensemble based proximity measure. We evaluate using two different damping factors $\beta=0.05$ and 0.005 . The results of using these two different damping factors are similar, and we only report the results using $\beta=0.05$ in the interest of brevity. Katz achieves both low false negative rate and low false positive rate. Katz is the best in Digg and MySpace datasets, and the second best in LiveJournal and Flickr. In YouTube dataset, Katz does not give a good

which is consistent with the ranking between direct proximity measures and sophisticated measures shown in Figure 8 and Figure 9. The ranking shows that as the high degree node's coverage increases, direct proximity measures (such as the number of common neighbors and shortest path distances) perform better than Katz and vice versa. 


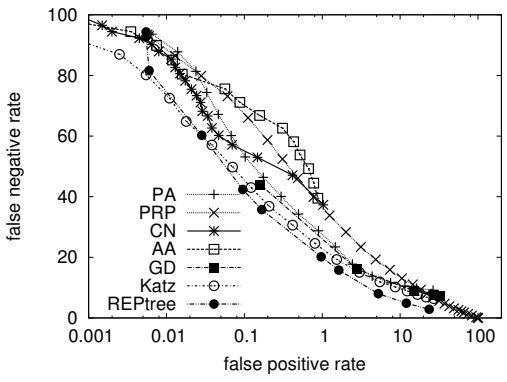

(a) Digg

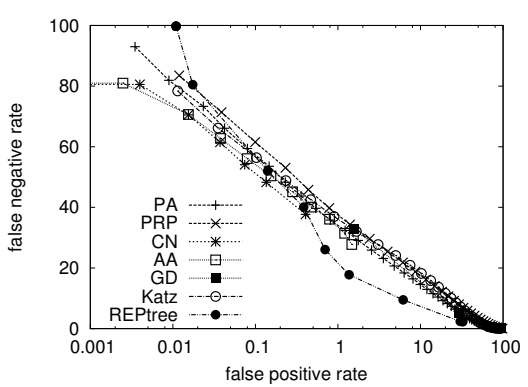

(d) MySpace

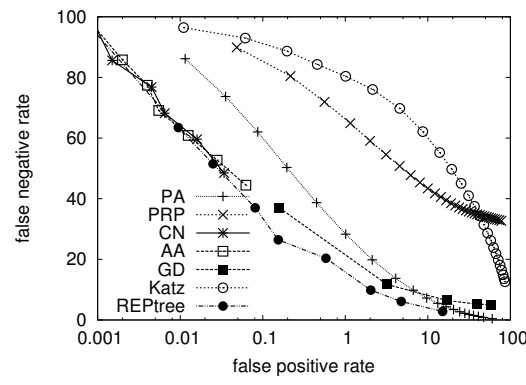

(b) Flickr

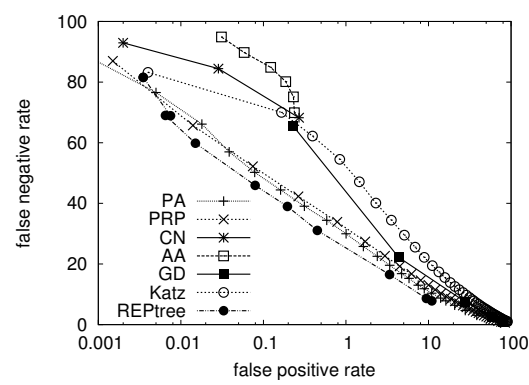

(e) YouTube

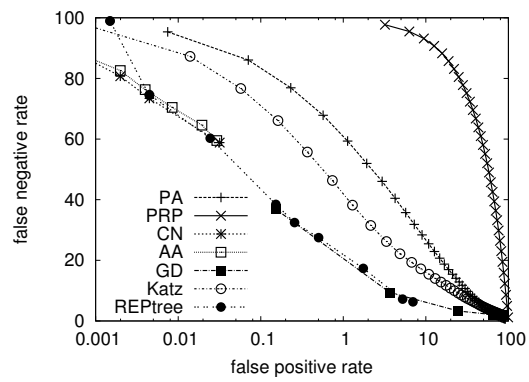

(c) LiveJournal

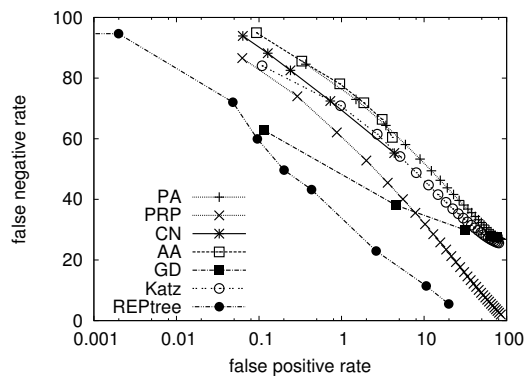

(f) Wikipedia

Figure 8: Link prediction accuracy for different online social networks

\subsubsection{Evaluation of Composite Link Predictor}

Next we combine multiple proximity measures to improve link prediction accuracy. When building a decision tree, at each node, REPtree chooses the best attribute (e.g., proximity measure) which splits samples into different classes (e.g., positive and negative) in the most effective way using information gain as criterion. To draw the entire accuracy trade-off curve, we vary the weights of positive and negative samples in the training set. When the weights of positive samples are large, the learner focuses more on positive samples in classification and tries to minimize false negatives; when the weights of negative samples are large, the learner focuses more on negative samples and tries to minimize false positives.

Figure 10 depicts examples of decision trees in Digg, Flickr, and Wikipedia (where the weights of positive:negative samples are $1: 100,1: 10$, and 1:100, respectively). The decision process starts from the root node, it drills down by examining the corresponding metric value until reaching one of leaf nodes.

Figure 8 shows the performance of decision tree in different online social networks. We observe that REPtree consistently achieves the best accuracy. Specifically, REPtree outperforms the best basic link predictors in Flickr, YouTube, and Wikipedia dataset. In Figure 8 (b) and (c), while the overall accuracy trade-off curve for REPtree is very close to the curve for shortest path distance, the composite link predictor provides much better resolution and fills in the intermediate points missed by the shortest path predictor. This is useful when we want to further classify possible friendship candidates with the same shortest path distance. Since the shortest path distance is integer-valued and is typically very small in social networks (due to the small-world effect [27]), it yields only coarsegrained control (e.g., depending on whether the threshold is 2 or 3 hops, a large number of node pairs will be classified as positive or negative at the same time).

\section{RELATED WORK}

Social networks. Traditional social networks have been widely studied by sociologists. Refer to [52] for a detailed review. Social networks have also found a wide range of applications in business (e.g., viral marketing [23], fraud detection [11]), information technology (e.g., improving Internet search [35]), computer networks (e.g., overlay network construction [45]), and cyber security (e.g., email spam mitigation [22], identity verification [56]).

The explosive growth of online social networks has attracted significant attention $[2,3,5,37]$. In particular, $[3,37]$ have conducted in-depth measurement-based studies of several online social networks and report the power-law, small-world, and scale-free properties of online social networks. Different from these studies, which analyze the general network topological structure, we focus on scalable proximity estimation and link prediction in online social networks.

Proximity measures. There is a rich body of literature on proximity measures (e.g., [1,26,28,30,31,38,47,48,50]). For example, [50] proposes escape probability as a useful measure of direction-aware proximity, which is closely related the rooted PageRank we consider. But their technique for computing escape probability can only scale to networks with tens of thousands of nodes. Recent works on proximity measures (e.g., $[28,48])$ either dismiss pathensemble based proximity measures due to their high computational cost or leave it as future work to compare with them.

Link prediction. [30,31] first define the link prediction problem for social networks. To predict links, they calculate ten proximity measures between node pairs of the graph. The nodes are ranked based on their scores, where node pairs with higher score are more likely to form a link. They measure the effectiveness of the different proximity measures in predicting links in five co-authorship networks. However, they are limited to relatively small networks with only about 5000 nodes. Moreover, they do not combine different measures to gain better link prediction accuracy.

Dimensionality reduction. A variety of dimensionality reduction techniques have been developed in the area of data stream computation (see [39] for a detailed survey). One powerful technique is sketch $[9,10,24,29]$, a probabilistic summary technique proposed for analyzing large streaming datasets. Sketches achieves dimensionality reduction using projections along random vectors. Our proximity sketch is closely related to the count-min sketch [10]. 


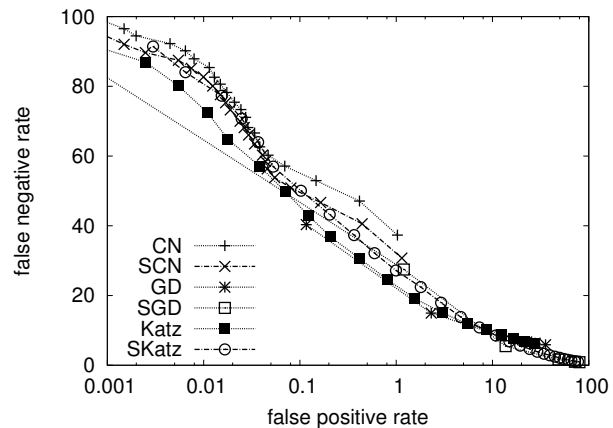

(a) Digg

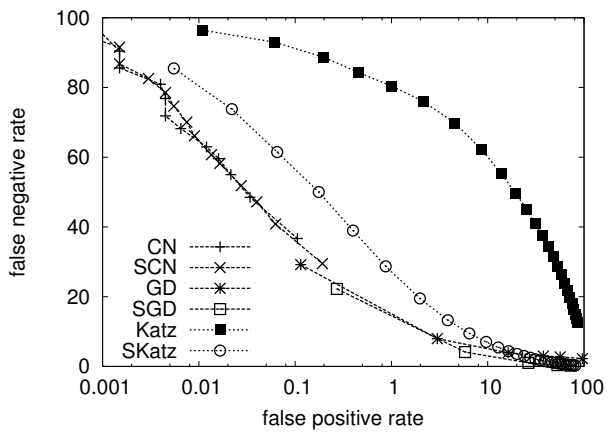

(c) Flickr

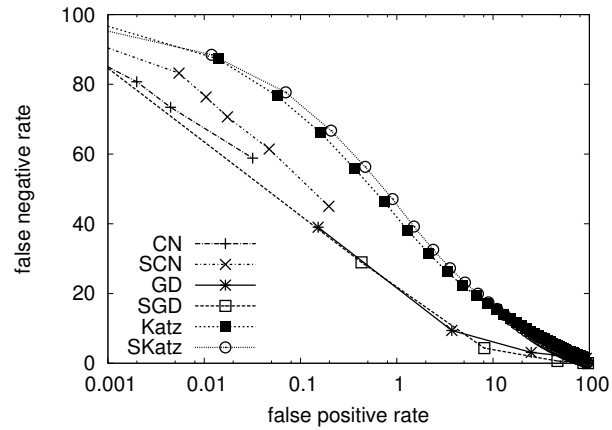

(b) LiveJournal

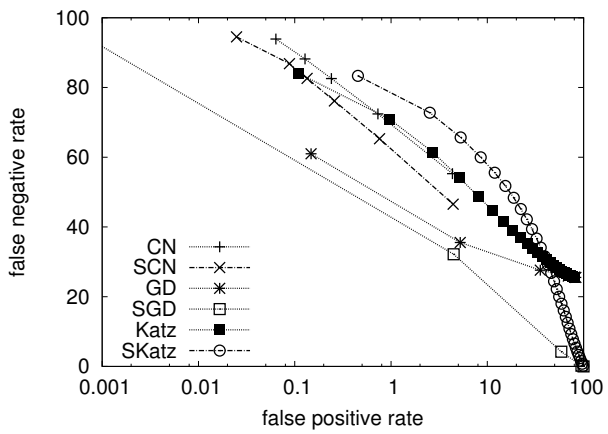

(d) Wikipedia

Figure 9: Link prediction accuracy with undirected edges. Note that edges in MySpace and YouTube are already undirected.

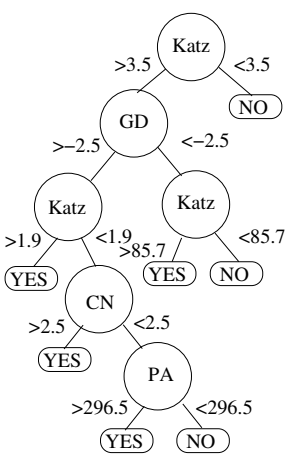

(a) Digg

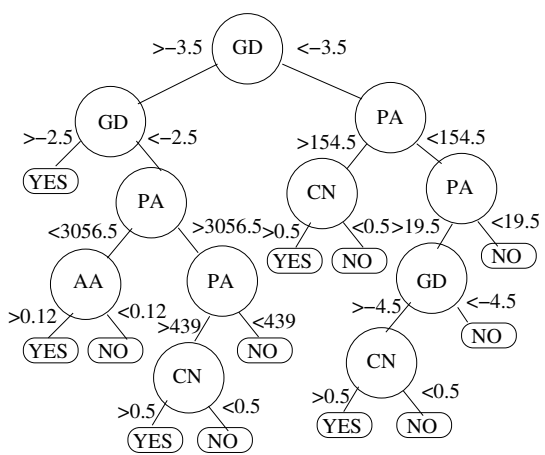

(b) Flickr

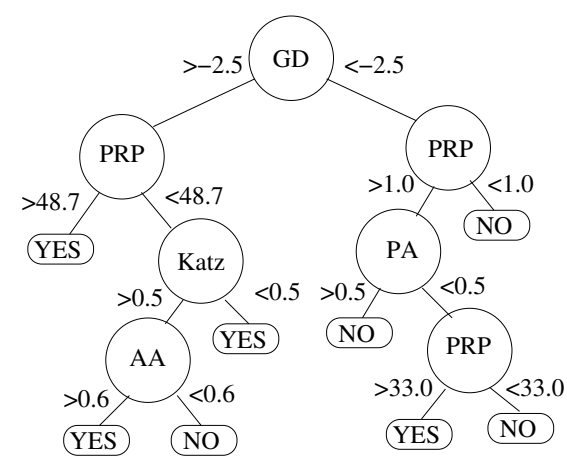

(c) Wikipedia

Figure 10: Examples of decision trees for link prediction

In computer networking, $\mathrm{Ng}$ and Zhang [43] propose the first network embedding technique, called Global Network Positioning (GNP), to infer end-to-end delay by embedding network delay in a low-dimensional Euclidean space. Several enhancements have been proposed since then [32, 34, 49]. In particular, [34] applies matrix factorization and can handle path asymmetry and violation of triangulation. To our knowledge, all these techniques have only been applied to networks with a few thousand nodes. Moreover, proximity is the opposite of distance - the lower the distance the higher the proximity. Thus, techniques for network distance embedding may not work well for proximity embedding.

\section{CONCLUSIONS}

In this paper, we develop several novel techniques to approximate a large family of proximity measures in massive, highly dynamic online social networks. Our techniques are accurate and can easily handle networks with millions of nodes, which are several orders of magnitude larger than what existing methods can sup- port. We then conduct extensive experiments on the effectiveness of a variety of proximity measures for predicting links in five popular online social networks. Our key new findings include: (i) the effectiveness of different proximity measures varies significantly across different networks and heavily depends on the fraction of edges contributed by the highest degree nodes, and (ii) combining multiple proximity measures consistently yields the best accuracy. In the future, we plan to leverage the insights we gain in this paper to design better proximity measures and more accurate link prediction algorithms.

\section{Acknowledgments}

This work was supported in part by NSF grants CNS-0546720 and CCF-0916309. We thank Inderjit Dhillon and the anonymous reviewers for their valuable feedback. We also thank Alan Mislove and Krishna Gummadi for sharing their online social network datasets. 


\section{REFERENCES}

[1] L. Adamic and E. Adar. Friends and neighbors on the web. Social Networks, 2003.

[2] L. A. Adamic, O. Buyukkokten, and E. Adar. A social network caught in the web. First Monday, 2003.

[3] Y.-Y. Ahn, S. Han, H. Kwak, S. Moon, and H. Jeong. Analysis of topological characteristics of huge online social networking services. In Proc. of WWW, 2007.

[4] Alexa global top 500 sites. http://www.alexa.com/site/ds/top_sites

[5] L. Backstrom, D. Huttenlocher, J. Kleinberg, and X. Lan. Group formation in large social networks: Membership, growth, and evolution. In Proc. of KDD, 2006.

[6] A. L. Barabasi, H. Jeong, Z. Néda, E. Ravasz, A. Schubert, and T. Vicsek. Evolution of the social network of scientific collaboration. Physica A: Statistical Mechanics and its Application, 2002.

[7] R. M. Bell, Y. Koren, and C. Volinsky. Chasing \$1,000,000: How we won the Netflix Progress Prize. Statistical Computing and Statistical Graphics Newsletter, 18(2):4-12, 2007.

[8] S. Chakrabarti. Dynamic personalized pagerank in entity-relation graphs. In Proc. of WWW, 2007.

[9] M. Charikar, K. Chen, and M. Farach-Colton. Finding frequent items in data streams. In Proc. of International Colloquium on Automata, Language and Programming (ICALP), 2002.

[10] G. Cormode and S. Muthukrishnan. An improved data stream summary: the count-min sketch and its applications. J. Algorithms, 2005.

[11] C. Cortes, D. Pregibon, and C. T. Volinsky. Communities of interest. Intelligent Data Analysis, 2002.

[12] K. Csalogány, D. Fogaras, B. Rácz, T. Sarlós, and P. File. Towards scaling fully personalized pagerank: Algorithms, lower bounds, and experiments. Internet Math, 2005.

[13] J. Davidsen, H. Ebel, and S. Bornholdt. Emergence of a small world from local interactions: Modeling acquaintance networks. Physical Review Letters, 2002.

[14] Digg. http://www.digg.com.

[15] Digg API. http://apidoc.digg.com.

[16] P. G. Doyle and J. L. Snell. Random walks and electric networks, Jan. 2000. [Online]. Available at

http://arxiv.org/abs/math/0001057v1.

[17] D. Epema, M. Livny, R. van Dantzig, X. Evers, and J. Pruyne. A worldwide flock of Condors: Load sharing among workstation clusters. Future Generation Computer Systems, 1996.

[18] Facebook. http://www. facebook.com.

[19] Facebook statistics. http: //www.facebook.com/press/info.php?statistics.

[20] Flickr. http://www.flickr.com.

[21] S. Garner. Weka: The waikato environment for knowledge analysis. In Proceedings of the New Zealand Computer Science Research Students Conference, pages 57-64, 1995. http://www.cs.waikato.ac.nz/ ml/weka/.

[22] S. Garriss, M. Kaminsky, M. J. Freedman, B. Karp, D. Mazieres, and H. Yu. RE: Reliable Email. In Proc. of Networked Systems Design and Implementation (NSDI), 2006.

[23] S. Hill, F. Provost, and C. Volinsky. Network-based marketing: Identifying likely adopters via consumer networks. Statistical Science, 2006.

[24] P. Indyk. Stable distributions, pseudorandom generators, embeddings and data stream computation. In Proc. of the 41 st Symposium on Foundations of Computer Science, 2000.

[25] E. M. Jin, M. Girvan, and M. E. J. Newman. The structure of growing social networks. Physical Review Letters, 2001.

[26] L. Katz. A new status index derived from sociometric analysis. Psychometrika, 1953.

[27] M. Kochen, editor. The Small World. Ablex, Norwood, NJ, 1989.
[28] Y. Koren, S. C. North, and C. Volinsky. Measuring and extracting proximity in networks. In Proc. of KDD, 2006.

[29] B. Krishnamurthy, S. Sen, Y. Zhang, and Y. Chen. Sketch-based change detection: Methods, evaluation, and applications. In Proc. of IMC, 2003.

[30] D. Liben-Nowell and J. Kleinberg. The link prediction problem for social networks. In Proc. of Conference on Information and Knowledge Management (CIKM), 2003.

[31] D. Liben-Nowell and J. Kleinberg. The link-prediction problem for social networks. J. Am. Soc. Inf. Sci. Technol., 2007.

[32] H. Lim, J. Hou, and C. H. Choi. Constructing Internet coordinate system based on delay measurement. In Proc. of IMC, 2003.

[33] LiveJournal. http://www.livejournal.com.

[34] Y. Mao and L. K. Saul. Modeling distances in large-scale networks by matrix factorization. In Proc. of IMC, New York, NY, USA, 2004

[35] A. Mislove, K. P. Gummadi, and P. Druschel. Exploiting social networks for Internet search. In Proc. of HotNets-V, 2006.

[36] A. Mislove, H. S. Koppula, K. Gummadi, P. Druschel, and B. Bhattacharjee. Growth of the flickr social network. In Proc. of SIGCOMM Workshop on Social Networks (WOSN), 2008.

[37] A. Mislove, M. Marcon, K. P. Gummadi, P. Druschel, and B. Bhattacharjee. Measurement and analysis of online social networks. In Proc. of IMC, 2007.

[38] T. Murata and S. Moriyasu. Link prediction of social networks based on weighted proximity measures. In Proc. of International Conference on Web Intelligence, Washington, DC, USA, 2007. IEEE Computer Society.

[39] S. Muthukrishnan. Data streams: Algorithms and applications. Foundations and Trends in Theoretical Computer Science, 2005.

[40] MySpace. http://www.myspace.com.

[41] MySpace 400 millionth user. http: //profile.myspace.com/index.cfm?fuseaction= user.viewprofiledfriendid $=400000000$.

[42] M. E. J. Newman. The structure and function of complex networks. SIAM Review, 2003.

[43] T. E. Ng and H. Zhang. Predicting internet network distance with coordinate-based appoaches. In Proc. of INFOCOMM, 2002.

[44] Nielson Online. Fastest growing social networks for September 2008. http: //blog.nielsen.com/nielsenwire/wp-content/ uploads/2008/10/press_release24.pdf.

[45] J. A. Patel, I. Gupta, and N. Contractor. JetStream: Achieving predictable gossip dissemination by leveraging social network principles. In Proc. of IEEE Network Computing and Applications (NCA), 2006.

[46] J. R. Quinlan. C4.5: programs for machine learning. Morgan Kaufmann Publishers Inc., San Francisco, CA, USA, 1993.

[47] M. Richardson and P. Domingos. The intelligent surfer: Probabilistic combination of link and content information in pagerank. In Proc. of NIPS, 2002.

[48] P. Sarkar, A. W. Moore, and A. Prakash. Fast incremental proximity search in large graphs. In Proc. of ICML, 2008.

[49] L. Tang and M. Crovella. Virtual landmarks for the Internet. In Proc. of IMC, 2003.

[50] H. Tong, C. Faloutsos, and Y. Koren. Fast direction-aware proximity for graph mining. In Proc. of KDD, 2007.

[51] M. V. Vieira, B. M. Fonseca, R. Damazio, P. B. Golgher, D. de Castro Reis, and B. Ribeiro-Neto. Efficient search ranking in social networks. In Proc. of CIKM, 2007.

[52] S. Wasserman and K. Faust. Social Networks Analysis: Methods and Applications. Cambridge University Press, Cambridge, UK, 1994.

[53] Wikipedia. Social network. http://en.wikipedia.org/wiki/Social_network.

[54] Wikipedia. http://www.wikipedia.org.

[55] YouTube. http://www. youtube.com.

[56] H. Yu, M. Kaminsky, P. B. Gibbons, and A. Flaxman. SybilGuard: Defending against Sybil attacks via social networks. In Proc. of SIGCOMM, 2006. 